\title{
Singular reduction of implicit Hamiltonian systems
}

\author{
Guido Blankenstein \\ Department of Mechanical Engineering \\ Katholieke Universiteit Leuven \\ Celestijnenlaan $300 \mathrm{~B}$ \\ B-3001 Leuven (Heverlee) \\ Belgium \\ Tudor S. Ratiu \\ Institut de Mathématiques Bernoulli \\ École Polytechnique Fédérale de Lausanne \\ MA-Ecublens, CH-1015 Lausanne \\ Switzerland
}

\begin{abstract}
This paper develops the reduction theory of implicit Hamiltonian systems admitting a symmetry group at a singular value of the momentum map. The results naturally extend those known for (explicit) Hamiltonian systems described by Poisson brackets.
\end{abstract}

keywords: implicit Hamiltonian systems, Dirac structures, symmetry, reduction

\section{Contents}

1 Introduction

2 Implicit Hamiltonian svstems

3 Symmetries and regular reduction

4 Singular reduction

5 Singular dynamics

6 Orbit type decomposition

7 Conclusions

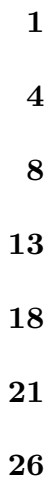

26

\section{Introduction}

Consider a symplectic manifold $(M, \omega)$ admitting a symmetry Lie group $G$ (with Lie algebra denoted by $\mathfrak{g}$ ) acting freely and properly on $M$, together with a corresponding equivariant momentum map $P: M \rightarrow \mathfrak{g}^{*}$. In [19] it is shown that, at a regular value $\mu \in \mathfrak{g}^{*}$ of the momentum map, the symplectic structure on $M$ naturally reduces to a symplectic structure $\omega_{\mu}$ on the reduced manifold $M_{\mu}=$ $P^{-1}(\mu) / G_{\mu}$, where $G_{\mu}$ is the coadjoint isotropy subgroup of $G$. Furthermore, the integral curves of a Hamiltonian vector field corresponding to a $G$-invariant Hamiltonian $H \in C^{\infty}(M)^{G}$ project to integral curves of the reduced Hamiltonian vector field corresponding to the reduced Hamiltonian $H_{\mu} \in C^{\infty}\left(M_{\mu}\right)$. This theory has been generalized in [17] to the case of Poisson manifolds: at a regular value $\mu$ of the momentum map, the Poisson bracket $\{\cdot, \cdot\}: C^{\infty}(M) \times C^{\infty}(M) \rightarrow C^{\infty}(M)$ on $M$ descends to a Poisson bracket $\{\cdot, \cdot\}_{\mu}$ on the reduced phase space $M_{\mu}$. Again, the Hamiltonian 
flow defined by a $G$-invariant Hamiltonian $H \in C^{\infty}(M)^{G}$ reduces to a Hamiltonian flow on $M_{\mu}$ corresponding to $H_{\mu} \in C^{\infty}\left(M_{\mu}\right)$. We refer to [1, 18, 22] for some excellent overviews of this theory.

Although by Sard's theorem regular reduction describes the "generic" case (i.e., the regular values of the momentum map are dense in $\mathfrak{g}^{*}$ ), interesting dynamics, such as bifurcation phenomena, occur at the singular values of the momentum map. Already in simple examples like the spherical pendulum moving with angular momentum zero (i.e., moving in a plane) and the Lagrange top (reducing the gravitational $S^{1}$ symmetry after regular reduction of the internal $S^{1}$ symmetry corresponding to the homogeneous mass distribution), one recognizes the need for the investigation of these special cases. This task has been taken up in [3, 4, 10, 11, 23, 24, 27, leading to the theory of so-called singular reduction of (symplectic and Poisson) Hamiltonian systems. See [9] for a nice overview and some worked out examples (including the spherical pendulum and the Lagrange top). The main difference with the regular reduction theory is that in the case of a singular value $\mu$ of the momentum map (i.e., the derivative, or tangent map, of $P$ at points of $P^{-1}(\mu)$ is not surjective), the reduced space $M_{\mu}$ is not a manifold. Therefore symplectic forms and (Hamiltonian) vector fields are not defined and, as a consequence, the reduced "Hamiltonian dynamics" cannot be written as a system of ordinary differential equations on $M_{\mu}$ (as in the regular case). However, since $M_{\mu}$ is still a topological space (relative to the natural quotient topology), a reduced Poisson bracket $\{\cdot, \cdot\}_{\mu}$ on the space of (Whitney) smooth functions $C^{\infty}\left(M_{\mu}\right)$ can still be defined. This bracket induces a Hamiltonian formalism that allows one to write the reduced Hamiltonian dynamics on the singular reduced space $M_{\mu}$. The Hamiltonian flow corresponding to this Hamiltonian dynamics is exactly the projection of the regular Hamiltonian flow on $M$. Finally, in [4, 9, 10, 11, 24, 27] it has been shown that the singular reduced space $M_{\mu}$ (resulting from a symplectic manifold $(M, \omega)$ ) may be stratified by symplectic manifolds, called pieces. The stratification is by orbit type decomposition. The Hamiltonian flow on $M_{\mu}$ leaves these pieces invariant and restricts to a (regular) Hamiltonian flow on each of the pieces.

Recently, 5, 6] have generalized the regular reduction theory for explicit Hamiltonian systems to a regular reduction theory for implicit Hamiltonian systems, extending preliminary work in [8] 28. Analogous to the symplectic form or the Poisson bracket in the classical theory, the underlying geometric structure of an implicit Hamiltonian system is that of a Dirac structure, defined as a maximally isotropic smooth vector subbundle of $T M \oplus T^{*} M$. This structure allows to define a Hamiltonian formalism generalizing the classical symplectic and Poisson formalisms by including the description of algebraic equations. That is, the "Hamiltonian dynamics" corresponding to a Dirac structure and a function $H \in C^{\infty}(M)$ consists of a set of differential and algebraic equations (and is therefore called implicit). Perhaps the most striking example of an implicit Hamiltonian system is that of a Hamiltonian system defined by a Poisson bracket on $M$, restricted to a submanifold of $M$ that is not the level set of a Casimir function (this example actually motivated the definition of a Dirac structure in [8] ). Using the notion of a Dirac structure introduced in [8, 13, implicit Hamiltonian systems were defined in [31, 32, 33, 20, 7] and successfully employed in the context of network modeling of energy conserving physical systems such as mechanical systems with (non)holonomic kinematic constraints, electrical LC circuits, electromechanical systems. We refer to [29] and references therein for more information; see also [5] for a detailed historical account. (Note that the Hamiltonian systems defined in [8, 13] are not truly implicit, but rather explicit systems.) The study of symmetries and reduction of implicit Hamiltonian systems evolved from 
preliminary results in [8, 13, 28, and led to a reduction theory for this class of systems described in [5] 6. There it was shown that a Dirac structure on $M$, admitting a symmetry Lie group $G$ with corresponding equivariant momentum map $P$, reduces to a Dirac structure on the reduced manifold $M_{\mu}$ if $\mu$ is a regular value of the momentum map. Furthermore, the (projectable) integral curves of the implicit system corresponding to a $G$-invariant function $H \in C^{\infty}(M)^{G}$ project to integral curves of the reduced implicit Hamiltonian system defined by the reduced Dirac structure and the reduced Hamiltonian $H_{\mu}$. The theory generalizes the classical regular reduction theory for symplectic and Poisson Hamiltonian systems, as well as the recently developed reduction theories for constrained mechanical systems. Section 3 briefly recalls the main results of [5] 6]; for a discussion of the connection with the reduction theory for constrained mechanical systems we refer to [5] [6].

The goal of this paper is to develop reduction theory for implicit Hamiltonian systems at singular values of the momentum map. We restrict our attention to a fairly general class of Dirac structures, described by a generalized Poisson bracket and a distribution of derivations (i.e., vector fields) on smooth functions. We consider the special subclass of symmetries that are symmetries of both the generalized Poisson bracket and the distribution. Using these ingredients, we prove that one can define a so-called topological Dirac structure on the singular reduced space $M_{\mu}$ (where for easiness of exposition we will take $\mu=0$ ), representing the reduced Dirac structure. This topological Dirac structure, whose construction implicitly uses the Sikorski differential spaces (see [11, 26]), defines a Hamiltonian formalism on the reduced space $M_{\mu}$ and the dynamics corresponding to an implicit Hamiltonian system with Hamiltonian $H \in C^{\infty}\left(M_{\mu}\right)$ are described. It is shown that the (projectable) integral curves of the implicit Hamiltonian system on $M$ corresponding to a $G$-invariant function $H \in C^{\infty}(M)^{G}$ project to "integral curves" of the Hamiltonian dynamics defined on the singular reduced space. The orbit type decomposition defines a stratification of the singular reduced space. It is shown that, under certain conditions, the topological Dirac structure restricts to regular reduced Dirac structures on the pieces (which are always manifolds) and that the Hamiltonian flow restricts to a regular Hamiltonian flow on each of these pieces.

The paper is organized as follows. Section 2 gives a brief introduction to Dirac structures and implicit Hamiltonian systems. The basic results concerning symmetries and regular reduction of implicit Hamiltonian systems are recalled in section 3 Section 4 describes in a purely topological way how to reduce an implicit Hamiltonian system admitting a symmetry group. The result is a so-called topological Dirac structure on the singular reduced space (in general, not a manifold). It is shown that if the symmetry group acts regularly and the value of the momentum map is regular, and hence the reduced space is a smooth manifold, then the singular reduced implicit Hamiltonian system equals the regular reduced implicit Hamiltonian system as defined in section 3 Section 5 discusses the dynamics of a singular reduced implicit Hamiltonian system. It is shown that the "projectable" solutions of the original system project to solutions of the singular reduced system. Section [6] describes the decomposition of the singular reduced space into smooth manifolds called pieces. The singular reduced implicit Hamiltonian system restricts to regular reduced implicit Hamiltonian systems on the pieces. Section 7 contains some concluding remarks. 


\section{Implicit Hamiltonian systems}

Let $M$ be a smooth $n$-dimensional manifold and let $T M \oplus T^{*} M$ denote the vector bundle whose fiber at $x \in M$ is $T_{x} M \times T_{x}^{*} M$. (Throughout this paper all geometric objects are assumed to be smooth, so when manifolds, vector bundles, sections are mentioned, they are all smooth.) A Dirac structure on $M$ is defined as follows.

Definition 1. A smooth vector subbundle $\mathcal{D} \subset T M \oplus T^{*} M$ is called a Dirac structure if for every fiber $\mathcal{D}(x) \subset T_{x} M \times T_{x}^{*} M, x \in M$, one has $\mathcal{D}(x)=\mathcal{D}^{\perp}(x)$, where

$$
\mathcal{D}^{\perp}(x)=\left\{\left(w, w^{*}\right) \in T_{x} M \times T_{x}^{*} M \mid\left\langle v^{*}, w\right\rangle+\left\langle w^{*}, v\right\rangle=0, \forall\left(v, v^{*}\right) \in \mathcal{D}(x)\right\} .
$$

(Here $\langle\cdot, \cdot\rangle$ denotes the duality pairing between $T M$ and $\left.T^{*} M.\right)$

Notice that $\mathcal{D}$ being a vector subbundle of $T M \oplus T^{*} M$ implies that its fibers all have the same dimension, i.e., $\operatorname{dim} \mathcal{D}(x)=\operatorname{dim} \mathcal{D}\left(x^{\prime}\right), \forall x, x^{\prime} \in M$. In particular, if $\mathcal{D}$ is a Dirac structure then $\operatorname{dim} \mathcal{D}(x)=n, \forall x \in M$. Furthermore, $\mathcal{D}(x)=\mathcal{D}^{\perp}(x), x \in M$, implies that

$$
\left\langle v^{*}, v\right\rangle=0, \forall\left(v, v^{*}\right) \in \mathcal{D}(x) .
$$

Remark 2. In [12] a constant Dirac structure on a vector space $\mathcal{V}$ is defined as a vector subspace $\mathcal{D} \subset \mathcal{V} \times \mathcal{V}^{*}$ such that $\mathcal{D}=\mathcal{D}^{\perp}$. An equivalent way of writing Definition 1 is therefore: a Dirac structure on a manifold $M$ is a smooth vector subbundle $\mathcal{D} \subset T M \oplus T^{*} M$ such that each fiber $\mathcal{D}(x), x \in M$, is a constant Dirac structure on $T_{x} M$.

In [8] there is yet another slightly different definition of a Dirac structure. Denote by $\mathfrak{X}_{l o c}(M)$ (respectively $\mathfrak{X}(M)$ ) the space of local (respectively global) smooth sections of $T M$, that is, these are the spaces of smooth local (respectively global) vector fields on $M$. Similarly, $\Omega_{l o c}^{k}(M)$ and $\Omega^{k}(M)$ denote the spaces of smooth local and global $k$-forms on $M$. The spaces of smooth local and global sections of the vector subbundle $\mathcal{D} \subset T M \oplus T^{*} M$ are denoted by $\mathfrak{D}_{l o c}$ and $\mathfrak{D}$ respectively. Throughout, let $X, Y \in \mathfrak{X}_{l o c}(M)$ and $\alpha, \beta \in \Omega_{l o c}^{1}(M)$. Define a pairing on smooth sections of $T M \oplus T^{*} M$ by

$$
\langle\langle(X, \alpha),(Y, \beta)\rangle\rangle=\langle\alpha, Y\rangle+\langle\beta, X\rangle \text {, for }(X, \alpha),(Y, \beta) \in T M \oplus T^{*} M .
$$

According to [8], a Dirac structure on $M$ is a smooth vector subbundle $\mathcal{D} \subset T M \oplus T^{*} M$ such that

1. $\mathcal{D}$ is isotropic, i.e., for every two (local) sections $(X, \alpha),(Y, \beta) \in \mathfrak{D}_{l o c}:\langle\langle(X, \alpha),(Y, \beta)\rangle\rangle=0$;

2. $\mathcal{D}$ is maximal, i.e., if $(Y, \beta)$ is a (local) section of $T M \oplus T^{*} M$ such that $\langle\langle(X, \alpha),(Y, \beta)\rangle\rangle=$ $0, \forall(X, \alpha) \in \mathfrak{D}_{l o c}$, then $(Y, \beta) \in \mathfrak{D}_{l o c}$.

It is easily shown that this definition given in [8] and Definition 1 are equivalent. Indeed, since $\mathcal{D}$ is a smooth vector subbundle, every $\left(v, v^{*}\right) \in \mathcal{D}(x)$ can be extended to a local section $(X, \alpha) \in \mathfrak{D}_{l o c}$. Furthermore, $\mathcal{D}$ being a smooth vector subbundle implies that also $\mathcal{D}^{\perp} \subset T M \oplus T^{*} M$ (with fibers $\left.\mathcal{D}^{\perp}(x)\right)$ is a smooth vector subbundle and therefore also every $\left(w, w^{*}\right) \in \mathcal{D}^{\perp}(x)$ can be extended to a local section $(Y, \beta)$ of $\mathcal{D}^{\perp}$. Elementary linear algebra shows that $\mathcal{D}$ is isotropic if and only if

$\mathcal{D} \subset \mathcal{D}^{\perp}$ and that maximal isotropy is equivalent to equality in this inclusion or to the fact that $\operatorname{dim} \mathcal{D}(x)=n$ for all $x \in M$ (see, e.g., [1], Section 5.3 for this type of argument). 
Corresponding to a Dirac structure a number of (co-)distributions may be identified. Recall that a distribution $\Delta$ on a manifold $M$ is a map which assigns to each $x \in M$ a linear subspace $\Delta(x)$ of the tangent space $T_{x} M . \quad \Delta$ is called a smooth distribution if around any point these subspaces are spanned by a set of smooth vector fields, i.e., there exist $X_{1}, \ldots, X_{k} \in \mathfrak{X}_{l o c}(M)$ such that $\Delta(x)=\operatorname{span}\left\{X_{1}(x), \ldots, X_{k}(x)\right\}$. The distribution $\Delta$ is called constant dimensional if the dimension of the linear subspace $\Delta(x) \subset T_{x} M$ does not depend on the point $x \in M$. Notice that if $\Delta$ is a smooth constant dimensional distribution on $M$, then it defines a smooth vector subbundle (also denoted by $\Delta$ ) of the tangent bundle $T M$, with fibers $\Delta(x), x \in M$. Analogously, a codistribution $\Gamma$ is defined as a map which assigns to each $x \in M$ a linear subspace $\Gamma(x)$ of the cotangent space $T_{x}^{*} M$. Smoothness and constant dimensionality are defined in the same way as for distributions. A smooth constant dimensional codistribution defines a smooth vector subbundle of the cotangent bundle $T^{*} M$.

Now, any Dirac structure $\mathcal{D}$ naturally defines a distribution $\Delta$, with fibers given by ${ }^{1}$

$$
\Delta(x):=\left\{X(x) \mid X \in \mathfrak{X}_{l o c}(M),(X, 0) \in \mathfrak{D}_{l o c}\right\}
$$

and a codistribution $\Gamma$, whose fibers are defined $b y^{2}$

$$
\Gamma(x):=\left\{\alpha(x) \mid \alpha \in \Omega_{l o c}^{1}(M), \exists X \in \mathfrak{X}_{l o c}(M),(X, \alpha) \in \mathfrak{D}_{l o c}\right\} .
$$

Since $\mathcal{D}$ is isotropic it follows that $\Delta(x) \subset \Gamma^{\circ}(x)$, where $\Gamma^{\circ}(x)$ denotes the annihilating vector subspace of $\Gamma(x)$ in $T_{x} M$, that is, $\Gamma^{\circ}(x)=\left\{v \in T_{x} M \mid\left\langle v^{*}, v\right\rangle=0, \forall v^{*} \in \Gamma(x)\right\}$. Equivalently, $\Gamma(x) \subset \Delta^{\circ}(x)$, where $\Delta^{\circ}(x)$ denotes the annihilating vector subspace of $\Delta(x)$ in $T_{x}^{*} M$, that is, $\Delta^{\circ}(x):=\left\{v^{*} \in T_{x}^{*} M \mid\left\langle v^{*}, v\right\rangle=0, \forall v \in \Delta(x)\right\}$. Furthermore, if $\Gamma$ is constant dimensional, and hence defines a vector subbundle of $T^{*} M$, it follows by maximal isotropy of $\mathcal{D}$ that $\Delta(x)=\Gamma^{\circ}(x)$ (equivalently, $\Gamma(x)=\Delta^{\circ}(x)$ ). Notice that in this case also $\Delta$ is constant dimensional and hence defines a vector subbundle of $T M$.

A special property of a Dirac structure is defined in the following

Definition 3. A Dirac structure $\mathcal{D}$ is called closed, or integrable, if for all (local) sections $\left(X_{1}, \alpha_{1}\right)$, $\left(X_{2}, \alpha_{2}\right),\left(X_{3}, \alpha_{3}\right) \in \mathfrak{D}_{l o c}$

$$
\left\langle L_{X_{1}} \alpha_{2}, X_{3}\right\rangle+\left\langle L_{X_{2}} \alpha_{3}, X_{1}\right\rangle+\left\langle L_{X_{3}} \alpha_{1}, X_{2}\right\rangle=0 .
$$

Equivalently [8, 13, 12], $\mathcal{D}$ is closed if and only if for all $\left(X_{1}, \alpha_{1}\right),\left(X_{2}, \alpha_{2}\right) \in \mathfrak{D}_{\text {loc }}$

$$
\left(\left[X_{1}, X_{2}\right], L_{X_{1}} \alpha_{2}-L_{X_{2}} \alpha_{1}+\mathrm{d}\left\langle\alpha_{1}, X_{2}\right\rangle\right) \in \mathfrak{D}_{l o c}
$$

The notation $L_{X}$ is reserved for the Lie derivative operator (acting on any type of tensor field) defined by the (local) vector field $X$ on $M$.

It is easy to see that the graph of a symplectic form $\omega: T M \rightarrow T^{*} M$ or the graph of the skewsymmetric vector bundle map $J: T^{*} M \rightarrow T M$ induced by a Poisson bracket on $M$ defines a Dirac

\footnotetext{
${ }^{1}$ In the literature on implicit Hamiltonian systems this distribution is usually denoted by $\mathrm{G}_{0}$ and sometimes called the characteristic distribution. However, in order to avoid confusion with notation defined later on in this paper we decided to adopt a different notation here.

${ }^{2}$ This codistribution is usually denoted by $\mathrm{P}_{1}$ in the literature.
} 
structure on $M$. As customary, we will call both the bundle map $J$ and the two-tensor defined by $\{\cdot, \cdot\}$ the Poisson structure on $M$. Closedness of $\mathcal{D}$ corresponds in these two cases to the condition that $\omega$ is a closed two-form, respectively, the Poisson bracket satisfies the Jacobi identity.

In this paper we will concentrate on a rather frequently occurring type of Dirac structure defined as follows. Let $\{\cdot, \cdot\}: C^{\infty}(M) \times C^{\infty}(M) \rightarrow C^{\infty}(M)$ be a generalized Poisson bracket on $M$. That is, $\{\cdot, \cdot\}$ is skew-symmetric, bilinear, and satisfies the Leibniz property. Denote the corresponding vector bundle map by $J: T^{*} M \rightarrow T M$ (i.e., $J(d H, d F)=\{H, F\}, H, F \in C^{\infty}(M)$ ); recall that $J$ is skew-symmetric. Note that we do not require $\{\cdot, \cdot\}$ to satisfy the Jacobi identity and neither that $J$ have constant rank. Moreover, given a subbundle $\Delta$ of $T M$ (i.e., a smooth constant dimensional distribution $\Delta$ on $M$ ), it is easy to see that the vector subbundle $\mathcal{D} \subset T M \oplus T^{*} M$ with fiber

$$
\mathcal{D}(x)=\left\{\left(v, v^{*}\right) \in T_{x} M \times T_{x}^{*} M \mid v-J(x) v^{*} \in \Delta(x), v^{*} \in \Delta^{\circ}(x)\right\}
$$

defines a Dirac structure on $M$. In terms of its local sections this is expressed as

$$
\mathfrak{D}_{l o c}=\left\{(X, \alpha) \in \mathfrak{X}_{l o c}(M) \oplus \Omega_{l o c}^{1}(M) \mid X-J \alpha \text { is a local section of } \Delta, \alpha \text { is local section of } \Delta^{\circ}\right\} \text {, }
$$

where $\Delta^{\circ}$ denotes the vector subbundle of $T^{*} M$ whose fiber at $x \in M$ equals $\Delta^{\circ}(x)$.

Remark 4. In 12 it is shown that, under a mild constant dimensionality assumption, every Dirac structure can be written in the form (2.8) or, equivalently, (2.9). Indeed, if $\mathcal{D}$ is an arbitrary Dirac structure on $M$, define the codistribution $\Gamma$ as in (2.5), and assume that $\Gamma$ is constant dimensional and hence defines a vector subbundle of $T^{*} M$. Then there exists a well defined (see [12]) skewsymmetric vector bundle map $J(x): \Gamma(x) \subset T_{x}^{*} M \rightarrow(\Gamma(x))^{*} \subset T_{x} M, x \in M$, defined by

$$
J(x) v^{*}=\bar{v} \in(\Gamma(x))^{*}, v^{*} \in \Gamma(x),
$$

where $\bar{v} \in(\Gamma(x))^{*}$ is the restriction of some $v \in T_{x} M$ to $\Gamma(x) \subset T_{x}^{*} M$ which satisfies the condition that $\left(v, v^{*}\right) \in \mathcal{D}(x)$. Notice that the kernel of $J(x)$ is given by the codistribution $\Gamma_{0}$ with fibers defined $\mathrm{by}^{3}$

$$
\Gamma_{0}(x):=\left\{\alpha(x) \mid \alpha \in \Omega_{l o c}^{1}(M),(0, \alpha) \in \mathfrak{D}_{l o c}\right\} .
$$

Then the Dirac structure $\mathcal{D}$ takes the form (2.8), or, equivalently, (2.9), with $\Delta=\Gamma^{\circ}$.

The map $J$ may be locally extended to a generalized Poisson structure $J: T^{*} M \rightarrow T M$.

Notice that, in general, the Dirac structure defined in (2.8) is not closed. Although closedness does not play an important role in the rest of the paper we remark for completeness that (2.9) defines a closed Dirac structure if and only if (see [12])

1. $\Delta$ is involutive,

2. the bracket $\{\cdot, \cdot\}$ restricted to the set of admissible functions $\mathcal{A}_{D}:=\left\{H \in C^{\infty}(M) \mid d H \in \Delta^{\circ}\right\}$ defines a Poisson bracket on $\mathcal{A}_{D}$ (that is, the Jacobi identity holds).

Remark 5. Before leaving our introduction to Dirac structures and moving on to the description of implicit Hamiltonian systems, we would like to mention the following generalization. In [15] a

\footnotetext{
${ }^{3}$ This codistribution is usually denoted by $\mathrm{P}_{0}$ in the literature.
} 
Dirac structure is defined as a maximal isotropic subbundle $\mathcal{D} \subset \mathcal{A} \oplus \mathcal{A}^{*}$, where the pair $\left(\mathcal{A}, \mathcal{A}^{*}\right)$ defines a Lie bialgebroid over a smooth manifold $M$. The isotropy is defined with respect to the natural pairing $\langle\langle\rangle$,$\rangle defined analogously as in (2.3) by \mathcal{A}$ and its dual $\mathcal{A}^{*}$. If we take the special case $\mathcal{A}=T M$ and dually $\mathcal{A}^{*}=T^{*} M$, then we are back at the definition given earlier in this paper. For more information on this generalization we refer to [15] and the references therein. We remark that [15] require the Dirac structure to be closed. In their terminology, Dirac structures are always closed, while Dirac structures not satisfying condition (2.7) are called almost Dirac structures. In this paper however, we prefer to use the terminology as introduced above, calling a maximally isotropic subbundle of $T M \oplus T^{*} M$ a Dirac structure and adding the prefix closed if (and only if) the conditions of Definition 3 are satisfied.

Now we turn to the definition of an implicit Hamiltonian system. Consider a Dirac structure $\mathcal{D}$ on $M$ and a smooth function $H \in C^{\infty}(M)$, called the Hamiltonian or energy function. Then the three-tuple $(M, \mathcal{D}, H)$ defines an implicit Hamiltonian system as follows:

Definition 6. The implicit Hamiltonian system $(M, \mathcal{D}, H)$ is defined as a set of smooth time functions $\left\{x(t) \mid x: \mathbb{R} \rightarrow M\right.$ of class $\left.C^{\infty}\right\}$ (called solutions) satisfying the condition

$$
(\dot{x}(t), d H(x(t))) \in \mathcal{D}(x(t)), \forall t .
$$

Equations (2.2) and (2.12) imply that implicit Hamiltonian systems are energy conserving, i.e.,

$$
\frac{d H}{d t}(x(t))=\langle d H(x(t)), \dot{x}(t)\rangle=0, \forall t .
$$

If $\mathcal{D}$ is the graph of a symplectic form $\omega$ or of a Poisson structure $J: T^{*} M \rightarrow T M$ then the above definition becomes that of a classical (explicit) symplectic or Poisson Hamiltonian system. On the other hand, if $\mathcal{D}$ is defined by (2.8) then the system includes the algebraic constraints

$$
d H(x(t)) \in \Delta^{\circ}(x(t)), \forall t
$$

Thus all solutions of the implicit Hamiltonian system necessarily lie in the constraint manifold

$$
M_{c}=\left\{x \in M \mid d H(x) \in \Delta^{\circ}(x)\right\}
$$

Since the implicit Hamiltonian system defines a set of differential and algebraic equations, there is not an existence and uniqueness result as one has for solutions of classical Hamiltonian systems described by ordinary differential equations. In particular, not every point $x_{0} \in M_{c}$ necessarily lies on the trajectory of some solution $x(t)$ of the system, and neither are the solutions through a point $x_{0} \in M_{c}$ (if existing) necessarily unique (this happens, for instance, if the Lagrange multipliers corresponding to the algebraic constraints cannot be solved uniquely). In the sequel we will not investigate these problems. Instead we will study the reduction of the underlying Dirac structure in the presence of symmetries (defined later on) and show that certain "projectable" solutions (if existing) will project to solutions of an implicit Hamiltonian system on the reduced space.

The problem of existence and uniqueness of solutions to implicit systems is an important and active area of research and will not be touched upon here. We would only like to mention the special case of so-called index 1 systems. Consider the implicit Hamiltonian system defined by 
the Dirac structure (2.8) and the Hamiltonian function $H \in C^{\infty}(M)$. Let the vector subbundle $\Delta$ be (locally) written as the span of the independent vector fields $g_{1}, \ldots, g_{m}$. Then the constraint manifold can be written as

$$
M_{c}=\left\{x \in M \mid L_{g_{j}} H(x)=0, j=1, \ldots, m\right\} .
$$

Now assume that the constraints are of index 1, that is, the matrix

$$
\left[L_{g_{i}} L_{g_{j}} H(x)\right]_{i, j=1, \ldots, m}
$$

is nonsingular for all $x \in M_{c}$. In that case one can restrict the implicit Hamiltonian system $(M, \mathcal{D}, H)$ to an explicit Hamiltonian system on $M_{c}$, defined by a (possibly nonintegrable) Poisson bracket on $M_{c}$, see [32, 5]. Its corresponding dynamics is thus given by a set of ordinary differential equations on $M_{c}$. Standard existence and uniqueness results now yield that through every point $x_{0} \in M_{c}$ there is a unique solution of the implicit Hamiltonian system (restricted to $M_{c}$ ).

Example 7. As an important example of implicit Hamiltonian systems we like to mention the class of mechanical systems with kinematic constraints. These systems are described by implicit Hamiltonian systems $(M, \mathcal{D}, H)$ with $\mathcal{D}$ of the form (2.8). Here, $H$ is the total energy of the system, (the sum of kinetic and potential energies for classical mechanical systems) and the phase space $M=T^{*} Q$ is the cotangent bundle of the configuration space $Q$; local coordinates are denoted, as usual, by $(q, p) \in T^{*} Q$. The Poisson bracket $\{\cdot, \cdot\}$ is the standard Poisson bracket corresponding to the canonical symplectic form $\omega=d p \wedge d q$ on $T^{*} Q$ (i.e., the associated Poisson structure $J$ is given by $\left.\omega^{-1}\right)$. Finally, if we assume the kinematic constraints to be linear in the velocities (e.g., non-slipping constraints of a rolling wheel) then they can be (locally) written in the form

$$
A^{T}(q) \dot{q}=A^{T}(q) \frac{\partial H}{\partial p}(q, p)=0 .
$$

By d'Alembert's principle, the constraints (2.18) generate constraint forces of the form $F_{c}=A(q) \lambda$, where $\lambda$ are the Lagrange multipliers. It follows that the distribution $\Delta$ is (locally) described by the image of the matrix

$$
\left[\begin{array}{c}
0 \\
A(q)
\end{array}\right] .
$$

If the kinetic energy is defined by a positive definite metric on $Q$, then the constraints are of index 1, i.e., the Lagrange multipliers $\lambda$ can be solved uniquely. In this case the constrained mechanical system on $T^{*} Q$ can be written as an unconstrained generalized Hamiltonian system on $M_{c}$. In 30] it is shown that the corresponding Poisson bracket on $M_{c}$ satisfies the Jacobi identity if and only if the kinematic constraints are holonomic.

\section{Symmetries and regular reduction}

In this section we recall some of the results in [5, 6] concerning symmetries and reduction of implicit Hamiltonian systems. These results act as a reference for the results obtained in this paper and will be specifically needed in section [6 to show that the singular reduced Dirac structure restricts to regular reduced Dirac structures on the pieces corresponding to the orbit type decomposition 
of the singular reduced space $M_{0}$. We refer to [5, 6] for a detailed treatment of symmetries and reduction of implicit Hamiltonian systems. We stress that, unless specifically stated otherwise, the results in this section are valid for arbitrary Dirac structures, not necessarily of the form (2.8).

Definition 8. A smooth vector field $Y$ on $M$ is called a symmetry of the Dirac structure $\mathcal{D}$ if for every (local) section $(X, \alpha)$ of $\mathcal{D}$, one also has that $\left(L_{Y} X, L_{Y} \alpha\right)$ is a (local) section of $\mathcal{D}$. $Y$ is called a symmetry of the implicit Hamiltonian system $(M, \mathcal{D}, H)$ if $Y$ is a symmetry of $\mathcal{D}$ and a symmetry of $H$, i.e., $L_{Y} H=0{ }^{4}$

The above notion of symmetry generalizes the classical notion of symmetry in symplectic or Poisson Hamiltonian systems. Indeed, if $\mathcal{D}$ is the graph of a symplectic form $\omega$ then $Y$ is a symmetry of $\mathcal{D}$ if and only if $L_{Y} \omega=0$. Likewise, if $\mathcal{D}$ is the graph of the skew-symmetric vector bundle map $J$ defining a Poisson structure on $M$, then $Y$ is a symmetry of $\mathcal{D}$ if and only if the integral flow of $Y$ is a Poisson map, i.e., $Y$ is a derivation of the Poisson bracket: $L_{Y}\left\{F_{1}, F_{2}\right\}=\left\{L_{Y} F_{1}, F_{2}\right\}+\left\{F_{1}, L_{Y} F_{2}\right\}$ for any $F_{1}, F_{2} \in C^{\infty}(M)$.

In the next sections we will consider a special subclass of symmetries defined by the following

Proposition 9. Consider a Dirac structure $\mathcal{D}$ of the type defined in (2.8). If the vector field $Y$ on $M$ is a derivation of the generalized Poisson bracket (equivalently, $L_{Y} J=0$ ) and $L_{Y} Z$ is a local section of $\Delta$ whenever $Z$ is a local section of $\Delta$, then $Y$ is a symmetry of $\mathcal{D}$.

In particular this means that we restrict our attention to the case where $Y$ is a symmetry of the generalized Poisson bracket as well as a symmetry of the vector subbundle $\Delta \subset T M$. These kinds of symmetries often arise in constrained mechanical systems, see also Remark 24 later on.

Specifically we will consider Lie algebra symmetries, defined as follows. Recall that a (left) Lie algebra action on a manifold $M$ is a Lie algebra anti-homomorphism $\xi \in \mathfrak{g} \mapsto \xi_{M} \in \mathfrak{X}(M)$ such that the map $(x, \xi) \in M \times \mathfrak{g} \mapsto \xi_{M}(x) \in T_{x} M$ is smooth. Then $\mathfrak{g}$ is a symmetry Lie algebra of $\mathcal{D}$ if $\xi_{M}$ is a symmetry of $\mathcal{D}$ for every $\xi \in \mathfrak{g}$. In particular, if the Dirac structure is of the type defined in (2.8), the criterion in Proposition 9 applies. Similarly, $\mathfrak{g}$ is a symmetry Lie algebra of an implicit Hamiltonian system $(M, \mathcal{D}, H)$ if each $\xi_{M}$ is a symmetry of $\mathcal{D}$ and also a symmetry of $H$, i.e., $L_{\xi_{M}} H=0$.

Lie algebra symmetries are often induced by Lie group actions. If $G$ is a Lie group with Lie algebra $\mathfrak{g}$ and $\phi: G \times M \rightarrow M$ is a smooth left action of $G$ on the manifold $M$, the infinitesimal generator of the action associated to $\xi \in \mathfrak{g}$ defined by

$$
\xi_{M}(x)=\left.\frac{d}{d t} \phi(\exp (\xi t), x)\right|_{t=0} \in T_{x} M, x \in M,
$$

induces a left Lie algebra action of $\mathfrak{g}$ on $M$. Then $G$ is said to be a symmetry Lie group of $\mathcal{D}$ if $\mathfrak{g}$ is a symmetry Lie algebra of $\mathcal{D}$. Similarly, $G$ is a symmetry Lie group of the implicit Hamiltonian system $(M, \mathcal{D}, H)$ if $\mathfrak{g}$ is a symmetry Lie algebra of this implicit Hamiltonian system.

We turn now to the analysis of the regular reduction process of Dirac structures and implicit Hamiltonian systems. We start by explaining how an implicit Hamiltonian system on $M$ can be restricted to an implicit Hamiltonian system on a submanifold $N$ of $M$. Let $\mathcal{D}$ be a Dirac structure on $M$ and let $N \subset M$ be a submanifold of $M$. Following [8] define for each $x \in N$ the map

\footnotetext{
${ }^{4}$ This is called a strong symmetry in 5$]$ 6].
} 
$\sigma(x): T_{x} N \times T_{x}^{*} M \rightarrow T_{x} N \times T_{x}^{*} N, x \in N$, by $\sigma(x)\left(v, v^{*}\right)=\left(v,\left.v^{*}\right|_{T_{x} N}\right)$, where $\left.v^{*}\right|_{T_{x} N}$ means the restriction of the covector $v^{*} \in T_{x}^{*} M$ to the subspace $T_{x} N \subset T_{x} M$. Define a vector subspace of $T_{x} N \times T_{x}^{*} N$ by

$$
\mathcal{D}_{N}(x)=\sigma(x)\left(\mathcal{D}(x) \cap\left(T_{x} N \times T_{x}^{*} M\right)\right), x \in N .
$$

It is clear that $\mathcal{D}_{N}(x) \subset \mathcal{D}_{N}^{\perp}(x), x \in N$. To prove the reverse inclusion, suppose that $\left(w, w^{*}\right) \in$ $\mathcal{D}_{N}^{\perp}(x) \subset T_{x} N \times T_{x}^{*} N$, i.e., $\left\langle v^{*}, w\right\rangle+\left\langle w^{*}, v\right\rangle=0, \forall\left(v, v^{*}\right) \in \mathcal{D}_{N}(x)$. Then $\left(v, v^{*}\right)=\sigma(x)\left(v, \bar{v}^{*}\right)$, where $\bar{v}^{*} \in T_{x}^{*} M,\left(v, \bar{v}^{*}\right) \in \mathcal{D}(x),\left.\bar{v}^{*}\right|_{T_{x} N}=v^{*}$ and since $v, w \in T_{x} N$, one gets

$$
0=\left\langle v^{*}, w\right\rangle+\left\langle w^{*}, v\right\rangle=\left\langle\bar{v}^{*}, w\right\rangle+\left\langle\bar{w}^{*}, v\right\rangle
$$

where $\bar{w}^{*}$ is an arbitrary extension of $w^{*}$ to $T_{x} M$. Since this relation holds for all $\left(v, \bar{v}^{*}\right) \in \mathcal{D}(x)$ with $v \in T_{x} N$, this implies that

$$
\begin{aligned}
\left(w, \bar{w}^{*}\right) & \in\left[\mathcal{D}(x) \cap\left(T_{x} N \times T_{x}^{*} M\right)\right]^{\perp}=\mathcal{D}^{\perp}(x)+\left(T_{x} N \times T_{x}^{*} M\right)^{\perp} \\
& =\mathcal{D}(x)+\left(\{0\} \times T_{x} N^{\circ}\right),
\end{aligned}
$$

so there exists a $\bar{u}^{*} \in T_{x} N^{\circ} \subset T_{x}^{*} M$ such that $\left(w, \bar{w}^{*}+\bar{u}^{*}\right) \in \mathcal{D}(x)$. However, since $w \in T_{x} N$ and $\sigma(x)\left(w, \bar{w}^{*}+\bar{u}^{*}\right)=\left(w,\left.\left(\bar{w}^{*}+\bar{u}^{*}\right)\right|_{T_{x} N}\right)=\left(w, w^{*}\right)$, it follows that $\left(w, w^{*}\right) \in \mathcal{D}_{N}(x)$, which shows that $\mathcal{D}_{N}^{\perp}(x) \subset \mathcal{D}_{N}(x)$. Assuming that the dimension of $\mathcal{D}(x) \cap\left(T_{x} N \times T_{x}^{*} M\right)$ is independent of $x \in N$, that is, that $\mathcal{D} \cap\left(T N \times T^{*} M\right)$ is a vector subbundle of $T N \times T^{*} M$, it follows that $\sigma$ is a vector bundle map and hence that $\mathcal{D}_{N}$ is a vector subbundle of $T N \times T^{*} N$. So we have proved (a slightly rewritten version of [8]):

Proposition 10. Consider a Dirac structure $\mathcal{D}$ on $M$ and let $N$ be a submanifold of $M$. Assume that $\mathcal{D}(x) \cap\left(T_{x} N \times T_{x}^{*} M\right), x \in N$, has constant dimension on $N$. Then the bundle $\mathcal{D}_{N}$ with fibers defined by (3.2) is a Dirac structure on $N$. This is called the restriction of $\mathcal{D}$ to $N$.

In order to do computations it is convenient to describe the restricted Dirac structure $\mathcal{D}_{N}$ in terms of its local sections. This gives the following proposition (an improved version of [5, 6] ). Let $\iota: N \hookrightarrow M$ denote the inclusion map.

Proposition 11. Consider a Dirac structure $\mathcal{D}$ on $M$ and let $N$ be a submanifold of $M$. Assume that $\mathcal{D}(x) \cap\left(T_{x} N \times T_{x}^{*} M\right), x \in N$, has constant dimension on $N$ and let $\mathcal{D}_{N}$ denote the restriction of $\mathcal{D}$ to $N$. Then $(\bar{X}, \bar{\alpha})$ is a local section of $\mathcal{D}_{N}$ if and only if there exists a local section $(X, \alpha)$ of $\mathcal{D}$ such that $\bar{X} \sim_{\iota} X$ and $\bar{\alpha}=\iota^{*} \alpha$. Otherwise stated, in terms of its local sections

$$
\left(\mathfrak{D}_{N}\right)_{l o c}=\left\{(\bar{X}, \bar{\alpha}) \in \mathfrak{X}_{l o c}(N) \oplus \Omega_{l o c}^{1}(N) \mid \exists(X, \alpha) \in \mathfrak{D}_{l o c} \text { such that } \bar{X} \sim_{\iota} X \text { and } \bar{\alpha}=\iota^{*} \alpha\right\} .
$$

Here $\bar{X} \sim_{\iota} X$ denotes the fact that $\bar{X}$ and $X$ are $\iota$-related and $\iota^{*}$ denotes the pullback by $\iota$. It can be shown that if $\mathcal{D}$ is closed, then also $\mathcal{D}_{N}$ is closed.

Now, let $(M, \mathcal{D}, H)$ be an implicit Hamiltonian system on $M$ and let $N$ be a submanifold of $M$ such that the constant dimension condition of Proposition 10 is satisfied and assume that (the flow corresponding to) the solutions of $(M, \mathcal{D}, H)$ leave the submanifold $N$ invariant. Restrict the Hamiltonian $H$ to a smooth function $H_{N}$ on $N$, i.e., $H_{N}=H \circ \iota$, and define the implicit Hamiltonian system $\left(N, \mathcal{D}_{N}, H_{N}\right)$ on $N$. Then: 
Proposition 12. Every solution $x(t)$ of $(M, \mathcal{D}, H)$ which is contained in $N$ is a solution of $\left(N, \mathcal{D}_{N}, H_{N}\right)$.

We remark that, in general, there is not a one-to-one correspondence between the solutions generated by the original system $(M, \mathcal{D}, H)$ and those generated by the restricted system $\left(N, \mathcal{D}_{N}, H_{N}\right)$. (Indeed, compare this with the case of restriction of a symplectic form $\omega$ on $M$ to an arbitrary submanifold $N$, leading to a nontrivial kernel for $\omega_{N}$.) In case $N$ happens to be the level set of a Casimir function of the Dirac structure $\mathcal{D}$ then there is a one-to-one correspondence between the solutions of the original system and of the restricted system; see [5] for more information.

Next, we explain how an implicit Hamiltonian system on $M$ admitting a symmetry Lie group $G$ can be projected to an implicit Hamiltonian system on the orbit space $M / G$. Consider a Dirac structure $\mathcal{D}$ on $M$ and let $G$ be a symmetry Lie group of $\mathcal{D}$, acting regularly on $M$, that is, the orbit space $M / G$ is a smooth manifold and the canonical projection map $\pi: M \rightarrow M / G$ is a surjective submersion. Let $V=\operatorname{ker} T \pi$ denote the vertical subbundle of $T M$, with fiber $V(x)=\operatorname{span}\left\{\xi_{M}(x) \mid \xi \in \mathfrak{g}\right\}$ for every $x \in M$. We assume that $V+\Delta$ is a smooth vector subbundle of $T M$, i.e., its fibers all have the same dimension. Furthermore, define the smooth vector subbundle $E \subset T M \oplus T^{*} M$ in terms of its local sections by

$$
\Gamma_{l o c}(E)=\left\{(X, \alpha) \in \mathfrak{X}_{l o c}(M) \oplus \Omega_{l o c}^{1}(M) \mid \alpha=\pi^{*} \hat{\alpha} \text { for some } \hat{\alpha} \in \Omega_{l o c}^{1}(M / G)\right\}
$$

(where $\Gamma_{\text {loc }}(E)$ is the space of local sections of the subbundle $E$ ) and assume that $\mathcal{D} \cap E$ is a smooth vector subbundle of $T M \oplus T^{*} M$, i.e., its fibers all have the same dimension. Then we have:

Proposition 13. [28, 6] Consider a Dirac structure $\mathcal{D}$ on $M$ admitting a symmetry Lie group $G$ acting regularly on $M$. Assume that $V+\Delta$ is a smooth vector subbundle of $T M$ and that $\mathcal{D} \cap E$ is a smooth vector subbundle of $T M \oplus T^{*} M$. Then $\mathcal{D}$ projects to a Dirac structure $\hat{\mathcal{D}}$ on $\hat{M}:=M / G$, described in terms of its local sections by

$$
\hat{\mathfrak{D}}_{l o c}=\left\{(\hat{X}, \hat{\alpha}) \in \mathfrak{X}_{l o c}(\hat{M}) \times \Omega_{l o c}^{1}(\hat{M}) \mid \exists(X, \alpha) \in \mathfrak{D}_{l o c} \text { such that } X \sim_{\pi} \hat{X} \text { and } \alpha=\pi^{*} \hat{\alpha}\right\} .
$$

$\hat{\mathcal{D}}$ is called the projection of $\mathcal{D}$ to $M / G$. Again, it can be shown that closedness of $\mathcal{D}$ implies closedness of $\hat{\mathcal{D}}$. Let $(M, \mathcal{D}, H)$ be an implicit Hamiltonian system admitting a symmetry Lie group acting regularly on $M$ such that the conditions in Proposition 13 are satisfied. The $G$-invariant function $H$ defines a function $\hat{H} \in C^{\infty}(M / G)$ by $H=\hat{H} \circ \pi$. Consider the implicit Hamiltonian system $(M / G, \hat{\mathcal{D}}, \hat{H})$. A $G$-projectable solution $x(t)$ of $(M, \mathcal{D}, H)$ is defined as a solution $x(t)$ of $(M, \mathcal{D}, H)$ for which there exists a projectable vector field $X \in \mathfrak{X}_{l o c}(M)$ (i.e., $X \sim_{\pi} \hat{X}$ for some $\left.\hat{X} \in \mathfrak{X}_{l o c}(M / G)\right)$ such that $\dot{x}(t)=X(x(t))$. The following proposition was obtained in [5, 6].

Proposition 14. If $x(t)$ is a $G$-projectable solution of $(M, \mathcal{D}, H)$ then $\hat{x}(t):=\pi(x(t))$ is a solution of $(M / G, \hat{\mathcal{D}}, \hat{H})$. Conversely, every solution $\hat{x}(t)$ of $(M / G, \hat{\mathcal{D}}, \hat{H})$ is locally the projection under $\pi$ of a $G$-projectable solution $x(t)$ of $(M, \mathcal{D}, H)$.

In [5] a simple example is given showing that not every solution of an implicit Hamiltonian system $(M, \mathcal{D}, H)$ admitting a symmetry Lie group $G$ is necessarily $G$-projectable. However, if the constraints are of index 1 , and therefore the implicit Hamiltonian system can be restricted to an explicit Hamiltonian system on the constraint manifold $M_{c}$, then it can be shown that every 
solution is $G$-projectable and therefore projects to a solution of the reduced implicit Hamiltonian system on $M / G$.

Finally, let us briefly recall some results in [5, 6] concerning the reduction of implicit Hamiltonian systems admitting a symmetry Lie group having an associated momentum map. Consider an implicit Hamiltonian system $(M, \mathcal{D}, H)$ admitting a symmetry Lie group $G$ with Lie algebra $\mathfrak{g}$. Assume that there exists an $A d^{*}$-equivariant map $P: M \rightarrow \mathfrak{g}^{*}\left(A d^{*}\right.$ denoting the coadjoint action), called momentum map, such that

$$
\left(\xi_{M}, d P_{\xi}\right) \in \mathfrak{D}, \forall \xi \in \mathfrak{g},
$$

where $P_{\xi} \in C^{\infty}(M)$ is defined by $P_{\xi}(x)=\langle P(x), \xi\rangle, x \in M$. Notice that if $\mathcal{D}$ is the graph of a symplectic form $\omega$ or a Poisson structure $J$, then this corresponds to the classical definition of a momentum map. Assuming that $\mu \in \mathfrak{g}^{*}$ is a regular value of $P$, it follows that the level set $P^{-1}(\mu)$ is a closed submanifold of $M$. Since the Hamiltonian is $G$-invariant, the solutions of $(M, \mathcal{D}, H)$ leave the level set $P^{-1}(\mu)$ invariant. Thus by (2.12), (3.7), and the identity $\mathcal{D}(x)=\mathcal{D}^{\perp}(x)$ it follows that

$$
\frac{d P_{\xi}}{d t}(x(t))=\left\langle d P_{\xi}(x(t)), \dot{x}(t)\right\rangle=-\left\langle d H, \xi_{M}\right\rangle(x(t))=0, \forall t, \forall \xi \in \mathfrak{g} .
$$

In other words, $P$ is a first integral of the implicit Hamiltonian system $(M, \mathcal{D}, H)$. Assuming that the conditions in Proposition 10 hold, we can restrict the implicit Hamiltonian system $(M, \mathcal{D}, H)$ to an implicit Hamiltonian system $\left(N, \mathcal{D}_{N}, H_{N}\right)$ on $N=P^{-1}(\mu)$. The system $\left(N, \mathcal{D}_{N}, H_{N}\right)$ admits the symmetry Lie group $G_{\mu}:=\left\{g \in G \mid A d_{g}^{*} \mu=\mu\right\}$. Assume that $G_{\mu}$ acts regularly on $N$, that is, $N / G_{\mu}$ is a smooth manifold with the canonical projection a surjective submersion, and assume that the conditions in Proposition 13 are satisfied. Then we can project the implicit Hamiltonian system $\left(N, \mathcal{D}_{N}, H_{N}\right)$ to an implicit Hamiltonian system $\left(M_{\mu}, \mathcal{D}_{\mu}, H_{\mu}\right)$, where $M_{\mu}=N / G_{\mu}=P^{-1}(\mu) / G_{\mu}$ is the regular reduced space and $H_{\mu} \in C^{\infty}\left(M_{\mu}\right)$ defined by $H_{\mu} \circ \pi=H_{N}$ is the reduced Hamiltonian. The implicit Hamiltonian system $\left(M_{\mu}, \mathcal{D}_{\mu}, H_{\mu}\right)$ is called the reduced implicit Hamiltonian system corresponding to $(M, \mathcal{D}, H)$ and $\mathcal{D}_{\mu}$ is called the reduced Dirac structure. If $\mathcal{D}$ is the graph of a symplectic form $\omega$, then $\mathcal{D}_{\mu}$ is precisely the graph of the Marsden-Weinstein reduced symplectic form $\omega_{\mu}$. Likewise, if $\mathcal{D}$ is the graph of a Poisson structure $J$ on $M$, then $\mathcal{D}_{\mu}$ is the graph of the reduced Poisson structure $J_{\mu}$ [17]. Notice, however, that contrary to the above mentioned classical reduction results, closedness of the Dirac structure is not required for the reduction scheme to work. This observation is important since it allows the reduction scheme to be applied to the class of mechanical systems with (possibly nonholonomic) kinematic constraints; see [5, 6] for further information and a discussion of its relationship to other recent results in this area. Of course, if $\mathcal{D}$ happens to be closed then also the reduced Dirac structure $\mathcal{D}_{\mu}$ will be closed.

Finally, we would like to mention that in [5, 6] it is shown that the reduction scheme can also be applied the other way around, starting with factorizing the symmetry group $G$ and afterwards restricting the result to a level set of the remaining first integrals (which actually turn out to be Casimir functions). The resulting implicit Hamiltonian system on $\tilde{M}_{\mu}$ is isomorphic to the system $\left(M_{\mu}, \mathcal{D}_{\mu}, H_{\mu}\right)$. Notice that $\tilde{M}_{\mu}$ is actually the orbit reduced space $P^{-1}\left(\mathcal{O}_{\mu}\right) / G$, where $\mathcal{O}_{\mu}$ denotes the coadjoint orbit in $\mathfrak{g}^{*}$ through $\mu$. See also [14, 16, 22] for some classical references.

Intrinsic reductions In this paragraph we will set the reduction results described above against what we will call intrinsic reductions. The latter are independent of any symmetry properties of 
the Dirac structure and, in fact, can be perfomed on any closed Dirac structure. These kind of reductions have been described in the literature by various authors [8, 13, 15, 5, 6].

Consider a closed Dirac structure $\mathcal{D}$ on $M$. Then by condition (2.7) it follows that the characteristic distribution $\Delta$ is involutive, and hence by Frobenius' Theorem defines a regular foliation $\Phi_{\Delta}$ of $M$ into integral submanifolds of $\Delta$. On the other hand, the distribution defined by ${ }^{5}$

$$
\Theta(x):=\left\{X(x) \mid X \in \mathfrak{X}_{l o c}(M), \exists \alpha \in \Omega_{l o c}^{1}(M),(X, \alpha) \in \mathfrak{D}_{l o c}\right\}
$$

clearly is also involutive, defining a regular foliation $\Phi_{\Theta}$ of $M$ into integral submanifolds of $\Theta$. (For the moment we will assume that both distributions are constant dimensional.)

There a two logical ways to "reduce" the Dirac structure on $M$ to a lower dimensional manifold. The first is to project the Dirac structure to the quotient manifold $M / \Phi_{\Delta}$, i.e., by factoring out the characteristic distribution. This was done in [8] where it was shown that the Dirac structure $\mathcal{D}$ on $M$ induces a well defined Poisson bracket on the quotient manifold $M / \Phi_{\Delta}$ ([8], Corollary 2.6.3). This remarkable result was generalized in [15] to Dirac structures on Lie bialgebroids as described in Remark [5] where is was refered to as Poisson reduction. In [5] it was observed that in fact this reduction can be seen as a special case of symmetry reduction if one notices that the distribution $\Delta$ is a symmetry distribution of $\mathcal{D}$, i.e., every vector field $Y \in \Delta$ is a symmetry of $\mathcal{D}$ as in Definition 8. The Dirac structure $\mathcal{D}$ can be projected to a Dirac structure $\hat{\mathcal{D}}$ on $M / \Phi_{\Delta}$ using Proposition 13. It turns out that $\hat{\mathcal{D}}$ is exactly the graph of the Poisson structure corresponding to the Poisson bracket defined by Courant [8]. We refer to [5], Example 4.2.4, p. 73, for more details.

The second reduction possibility is to restrict the Dirac structure to each of the integral submanifolds of $\Theta$. This can be done using Proposition 10 and results in a Dirac structure on each of the integral submanifolds of $\Theta$. In [5, 6] it is shown that each of the reduced Dirac structures represents a presymplectic structure on the corresponding leaf of the foliation, see [5], Example 4.1.8, p. 69, and [6], Example 9, p. 79. This corresponds to Theorem 2.3.6 in Courant [8] and Theorem 2.2 in Dorfman [13, stating that a closed Dirac structure has a foliation by presymplectic leaves.

Once more we want to stress that the reductions described above are "intrinsic" and have nothing to do with the existence of any symmetry groups of the implicit Hamiltonian system (although, as explained above, the first reduction can be interpreted in terms of symmetries of the Dirac structure). They can be perfomed on any closed Dirac structure. We will not concentrate on these intrinsic reductions anymore, and instead will investigate symmetry Lie groups of implicit Hamiltonian systems, together with their (singular) reductions. Doing so, we do not assume that the Dirac structure is closed and in fact all our results will be presented for the general case.

\section{Singular reduction}

Contrary to the regular reduction reviewed in the previous section we now describe in a purely topological way how to obtain a reduced Dirac structure on the reduced space $M_{\mu}$ if $M_{\mu}$ is not a manifold. This is the case when $\mu$ is a singular value of the momentum map $P$. In that case, vector fields and differential one-forms on $M_{\mu}$ are not defined and therefore the results described in the previous section cannot be used. Describing the dynamics corresponding to such a topologically

\footnotetext{
${ }^{5}$ This distribution is usually denoted by $\mathrm{G}_{1}$ in the literature.
} 
reduced Dirac structure on $M_{\mu}$ will be done in section 5. For easiness of exposition we will take $\mu=0$ throughout the rest of this paper.

From this point on we specifically consider only Dirac structures of the form (2.8), admitting symmetries as described in Proposition 9, that is, given a vector subbundle $\Delta \subset T M$, and a generalized Poisson structure $J: T^{*} M \rightarrow T M$ (the Jacobi identity does not necessarily hold), the Dirac structure is defined by

$$
\mathcal{D}(x)=\left\{\left(v, v^{*}\right) \in T_{x} M \times T_{x}^{*} M \mid v-J(x) v^{*} \in \Delta(x), v^{*} \in \Delta^{\circ}(x)\right\}
$$

and $Y \in \mathfrak{X}(M)$ is a symmetry of $\mathcal{D}$ if

$$
L_{Y} J=0 \text { and } L_{Y} Z \text { is a local section of } \Delta \text { whenever } Z \text { is a local section of } \Delta .
$$

Consider such a Dirac structure $\mathcal{D}$ on a manifold $M$ admitting a symmetry Lie group $G$ with corresponding $A d^{*}$-equivariant momentum map $P$ satisfying (3.7); it is not assumed that $G$ acts regularly on $M$. Let $\mu=0 \in \mathfrak{g}^{*}$ be a singular value of $P$ and consider the level set $N=P^{-1}(0)$ which is not a smooth submanifold of $M$. However, $N$ is a closed subset of $M$ and is a topological space relative to the induced subspace topology. The level set $N$ is $G$-invariant so one can endow the orbit space $M_{0}:=N / G=P^{-1}(0) / G$ with the quotient topology. Denote by $\pi: N \rightarrow M_{0}$ the canonical projection map, that is, $\pi$ maps $x \in N$ onto its orbit $G \cdot x \in M_{0}$.

Define the set of smooth functions on $M_{0}$ as follows (see [9], or for the original source see [25]).

Definition 15. A continuous function $f_{0}$ on $M_{0}$ is called smooth, denoted by $f_{0} \in C^{\infty}\left(M_{0}\right)$, if there exists a smooth $G$-invariant function $f \in C^{\infty}(M)^{G}$ such that $f_{0} \circ \pi=\left.f\right|_{P^{-1}(0)}$.

Given the singular reduced space $M_{0}$ together with its topology and a set of smooth functions $C^{\infty}\left(M_{0}\right)$ on $M_{0}$, we want to define a reduced Dirac structure on $M_{0}$. Let $\mathcal{D}$ be a Dirac structure on $M$ of the type defined in (4.1) and assume that the infinitesimal generators $\xi_{M}, \xi \in \mathfrak{g}$, satisfy the conditions in (4.2). Since $G$ is a symmetry Lie group of the generalized Poisson bracket $\{\cdot, \cdot\}$ : $C^{\infty}(M) \times C^{\infty}(M) \rightarrow C^{\infty}(M)$, corresponding to the bundle map $J$, we can use the theory in [3, 9] to define a generalized Poisson bracket $\{\cdot, \cdot\}_{0}: C^{\infty}\left(M_{0}\right) \times C^{\infty}\left(M_{0}\right) \rightarrow C^{\infty}\left(M_{0}\right)$ on the singular reduced space $M_{0}$. This goes as follows. Let $f_{0}, h_{0} \in C^{\infty}\left(M_{0}\right)$ and let $f, h \in C^{\infty}(M)^{G}$ be such that $f_{0} \circ \pi=\left.f\right|_{P^{-1}(0)}$ and $h_{0} \circ \pi=\left.h\right|_{P^{-1}(0)}$. Define the singular reduced generalized bracket by

$$
\left\{f_{0}, h_{0}\right\}_{0} \circ \pi=\left.\{f, h\}\right|_{P^{-1}(0)} .
$$

This gives a well defined generalized Poisson bracket on $M_{0}$. In particular, (4.3) does not depend on the choice of the $G$-invariant extensions $f$ and $h$ (whose existence is assumed, by definition).

Remark 16. The reduction theory in [3, 9] is only developed for the singular reduction of symplectic manifolds under a symmetry Lie group action. That is, the Poisson bracket $\{\cdot, \cdot\}$ is assumed to be nondegenerate and to satisfy the Jacobi identity. In principle, however, these results generalize immediately to the case of singular reduction of generalized Poisson brackets, as described above by (4.3). In particular, 3, 9, show that (under the assumption that $G$ acts properly) nondegeneracy of $\{\cdot, \cdot\}$ implies that of $\{\cdot, \cdot\}_{0}$. Also, from (4.3) it follows immediately that $\{\cdot, \cdot\}_{0}$ satisfies the Jacobi identity if $\{\cdot, \cdot\}$ does.

Once again for clarity: In this paper we do neither assume that the generalized Poisson bracket $\{\cdot, \cdot\}$ is nondegenerate, nor that it satisfies the Jacobi identity. Furthermore, properness of the group action is not assumed until Section 6 
Next, consider the vector subbundle $\Delta \subset T M$, defining a constant dimensional distribution on $M$. We show that $\Delta$ defines a vector space $\hat{\Delta}$ consisting of derivations on the space $C^{\infty}\left(M_{0}\right)$ of smooth function on $M_{0}$. Denote by $\Gamma_{\text {loc }}(\Delta)$ the local sections of the subbundle $\Delta \subset T M$. We show that every vector field $X \in \Gamma_{l o c}(\Delta)$ is "tangent" to $N$. In the regular case, when $N=P^{-1}(\mu)$ is a smooth submanifold of $M$, this means that $X$ restricts to a well defined vector field $\bar{X}$ on $N$. However, if $\mu=0$ is a singular value of the momentum map, then $N$ is not a smooth manifold and hence we have to define what "tangent" means. Recall that a vector field $X \in \mathfrak{X}(M)$ is in one-to-one correspondence with a derivation, also denoted by $X: C^{\infty}(M) \rightarrow C^{\infty}(M)$, on the set of smooth functions on $M$. The correspondence is given by the formula ${ }^{6}$

$$
X[f]=\langle d f, X\rangle, \forall f \in C^{\infty}(M) .
$$

A derivation $X$ on $M$ is said to be tangent to $N$ if it restricts to a well defined derivation $\bar{X}$ on the set of Whitney smooth functions on $N$. A continuous function $\bar{f}$ on $N$ is said to be a Whitney smooth function if there exists a smooth function $f$ on $M$ such that $\bar{f}=\left.f\right|_{N}$; the set of Whitney smooth functions on $N$ is denoted by $W^{\infty}(N)$. Otherwise stated, $X$ is tangent to $N$ if there exists a derivation $\bar{X}$ on $W^{\infty}(N)$ such that $X[f](x)=\bar{X}\left[\left.f\right|_{N}\right](x), \forall x \in N$, for all $f \in C^{\infty}(M)$. A necessary and sufficient condition for $X$ to be tangent to $N$ is that

$$
X[f](x)=X[h](x), \forall x \in N,
$$

for all $f, h \in C^{\infty}(M)$ such that $\left.f\right|_{N}=\left.h\right|_{N}$. Notice that in case $N$ is a smooth submanifold of $M$ and $N$ is closed in $M$, then the set $W^{\infty}(N)$ of Whitney smooth functions on $N$ is equal to the set $C^{\infty}(N)$ of all smooth functions on $N$ (as defined by the differential structure on the submanifold $N)$, and the above given definition yields the usual meaning of a vector field being tangent to the submanifold $N$ (and consequently restricting to a vector field on $N$ ).

Consider a vector field (or equivalently, derivation) $X$ on $M$, and define $\gamma(t)$ to be an integral curve of $X$ through $x_{0} \in M$ if $^{7}$

$$
\frac{d}{d t} f(\gamma(t))=X[f](\gamma(t)), \quad \forall t, \forall f \in C^{\infty}(M), \gamma(0)=x_{0} .
$$

Now, let $X \in \Gamma_{l o c}(\Delta)$ and $\gamma(t)$ be an integral curve of $X$ through $x_{0} \in P^{-1}(0)$. In particular,

$$
\frac{d}{d t} P_{\xi}(\gamma(t))=X\left[P_{\xi}\right](\gamma(t))=0, \forall t, \forall \xi \in \mathfrak{g}
$$

since by (2.8) (or (4.1)) and (3.7), $d P_{\xi}(x) \in \Delta^{\circ}(x), \forall x \in M$. This implies that the integral curve of $X \in \Gamma_{\text {loc }}(\Delta)$ through every $x_{0} \in P^{-1}(0)$ is contained in $P^{-1}(0)$ (conservation of the momentum map). Then by the equivalence of derivations and velocity vectors (remember that $M$ is a smooth manifold) it follows that

$$
X[f]\left(x_{0}\right)=\left.\frac{d}{d t} f(\gamma(t))\right|_{t=0}=\left.\frac{d}{d t} h(\gamma(t))\right|_{t=0}=X[h]\left(x_{0}\right),
$$

for all $f, h \in C^{\infty}(M)$ such that $\left.f\right|_{N}=\left.h\right|_{N}$. So we have shown that every vector field $X \in \Gamma_{l o c}(\Delta)$ is tangent to $N=P^{-1}(0)$ and consequently restricts to a well defined derivation $\bar{X}$ on $W^{\infty}(N)$.

\footnotetext{
${ }^{6}$ The derivation is usually called Lie derivative and is also denoted by $X[f]=L_{X} f$.

${ }^{7}$ Take the coordinate functions $f=x^{i}$ to obtain the usual definition $\dot{\gamma}^{i}(t)=X^{i}(\gamma(t))$.
} 
In conclusion, the constant dimensional distribution $\Delta$ on $M$ restricts to a vector space $\bar{\Delta}$ of derivations on $W^{\infty}(N)$. If $\Delta$ is locally spanned by the independent vector fields $X_{1}, \ldots, X_{m}$, then $\bar{\Delta}$ is locally spanned by the independent derivations $\bar{X}_{1}, \ldots, \bar{X}_{m}$.

Using the above results we are able to show that the distribution $\Delta$ on $M$ projects to a well defined vector space $\hat{\Delta}$ of derivations on the smooth functions $C^{\infty}\left(M_{0}\right)$. A vector field $X$ on $M$ is said to project to $M_{0}$ if there exists a derivation $\hat{X}$ on $C^{\infty}\left(M_{0}\right)$ such that for every $f \in C^{\infty}(M)^{G}$, $X[f](x)=\hat{X}\left[f_{0}\right](\pi(x)), \forall x \in N$, with $f_{0}$ defined by $f_{0} \circ \pi=\left.f\right|_{N}$. It is clear that $X$ restricts to a well defined derivation $\hat{X}$ on $C^{\infty}\left(M_{0}\right)$ if and only if $X[f](x)$ does not depend on the extension of $f_{0} \circ \pi$ off $N$ to $M$ and furthermore $X[f](x)=X[f](y)$ for all $x, y \in N$ such that $\pi(x)=\pi(y)$. Now let $X$ be a local section of $\Delta$. Since $X$ is tangent to $N$ it follows that $X[f](x)=\bar{X}\left[\left.f\right|_{N}\right](x)=$ $\bar{X}\left[f_{0} \circ \pi\right](x), \forall x \in N$, and therefore its value does not depend on the extension of $f_{0} \circ \pi$ off $N$ to $M$. It remains to show that

$$
X[f](x)=X[f](y), \forall x, y \in N \text { such that } \pi(x)=\pi(y)
$$

In general, this is not true for every local section $X$ of $\Delta$. However, we will show that there exists a basis of local sections $X_{1}, \ldots, X_{m}$ of $\Delta$ which satisfies (4.9).

Denote by $\mathfrak{V}_{l o c}$ the space of local sections of the vertical distribution $V$ defined by $V(x):=$ span $\left\{\xi_{M}(x) \mid \xi \in \mathfrak{g}\right\}$. Since $L_{\xi_{M}} \Gamma_{l o c}(\Delta) \subset \Gamma_{l o c}(\Delta)$ for every $\xi \in \mathfrak{g}$ by (4.2), it follows that $\left[\Gamma_{l o c}(\Delta), \mathfrak{V}_{l o c}\right] \subset \mathfrak{V}_{l o c}+\Gamma_{l o c}(\Delta)$. Indeed, taking an arbitrary local section of $V$ of the form $Y=$ $\sum_{i} h_{i} \xi_{M}^{i}, h_{i} \in C^{\infty}(M), i=1, \ldots, r=\operatorname{dim} \mathfrak{g}, \xi_{M}^{1}, \ldots, \xi_{M}^{r}$ being a local basis of $V$, and letting $X \in \Gamma_{l o c}(\Delta)$, it follows that

$$
[X, Y]=\left[X, \sum_{i=1}^{r} h_{i} \xi_{M}^{i}\right]=\sum_{i=1}^{r} h_{i}\left[X, \xi_{M}^{i}\right]+\left(L_{X} h_{i}\right) \xi_{M}^{i} \in \Gamma_{l o c}(\Delta)+\mathfrak{V}_{l o c}
$$

which proves the inclusion $\left[\Gamma_{l o c}(\Delta), \mathfrak{V}_{l o c}\right] \subset \mathfrak{V}_{l o c}+\Gamma_{l o c}(\Delta)$.

Assuming that the distribution $V+\Delta$ has constant dimension on $M$, the above inclusion implies that there exists a basis $X_{1}, \ldots, X_{m}$ of local sections of $\Delta$ such that $\left[X_{i}, \mathfrak{V}_{l o c}\right] \subset \mathfrak{V}_{l o c}, i=1, \ldots, m$; see e.g. Theorem 7.5 on page 214 in the book by Nijmeijer and van der Schaft [21] (in the notation of that theorem: the involutive distribution $D$ is $V$, the distribution $G$ is $\Delta$, and one takes $f=0$ ). In particular, $\left[X_{i}, \xi_{M}\right] \in \mathfrak{V}_{l o c}$, which implies that for all $f \in C^{\infty}(M)^{G}$

$$
0=\left[X_{i}, \xi_{M}\right][f]=X_{i}\left[L_{\xi_{M}} f\right]-L_{\xi_{M}}\left(X_{i}[f]\right)=-L_{\xi_{M}}\left(X_{i}[f]\right), \forall \xi \in \mathfrak{g} .
$$

This means that the function $X_{i}[f]$ is $G$-invariant and therefore satisfies (4.9). In conclusion, there exists a basis $X_{1}, \ldots, X_{m}$ of local sections of $\Delta$ such that each $X_{i}$ projects to a well defined derivation $\hat{X}_{i}$ on $C^{\infty}\left(M_{0}\right)$. The derivations $\hat{X}_{1}, \ldots, \hat{X}_{m}$ locally span (in other words, form a basis of) a vector space of derivations on $C^{\infty}\left(M_{0}\right)$, denoted by $\hat{\Delta}$.

Remark 17. In the regular case, i.e., when $\mu=0$ is a regular value of the momentum map and $G$ acts freely and properly on $M$, the reduced space $M_{0}$ is a smooth manifold. Furthermore, the set of smooth functions $C^{\infty}\left(M_{0}\right)$ equals the set of smooth functions as defined by the differential structure on $M_{0}$. Indeed, since $N=P^{-1}(0)$ is closed in $M$, every smooth $G$-invariant function on $N$ can be smoothly extended to a $G$-invariant function on $M[3]$. In that case the notion of a "projecting derivation" as defined above has the usual meaning of projection of a vector field on 
$M$ to a vector field on the reduced space $M_{0}$. In particular there exists a basis $X_{1}, \ldots, X_{m}$ of local sections of $\Delta$, which are tangent to $N$, such that the restrictions $\bar{X}_{1}, \ldots, \bar{X}_{m}$ project to $M_{0}$. That is, each $\bar{X}_{i}$ is $\pi$-related to a vector field $\hat{X}_{i}$ on $M_{0}$. The projected vector fields $\hat{X}_{1}, \ldots, \hat{X}_{m}$ form a basis of local sections of $\hat{\Delta}$.

So far we have defined a set of smooth functions $C^{\infty}\left(M_{0}\right)$ on the singular reduced space $M_{0}$, together with a generalized Poisson bracket $\{\cdot, \cdot\}_{0}$ and a vector space $\hat{\Delta}$ of derivations on $C^{\infty}\left(M_{0}\right)$. Recall that the original Dirac structure $\mathcal{D}$ (of the type given by (2.8) or (4.1) ) on the manifold $M$ was completely determined by the generalized Poisson bracket $\{\cdot, \cdot\}$, corresponding to $J$, and the distribution $\Delta$. Therefore it makes sense to define a reduced Dirac structure on $M_{0}$ as follows:

Definition 18. Consider the singular reduced space $M_{0}$ together with the set of smooth functions $C^{\infty}\left(M_{0}\right)$. The singular reduced Dirac structure $\mathcal{D}_{0}$ is defined as the pair $\left(\{\cdot, \cdot\}_{0}, \hat{\Delta}\right)$.

We also call $\mathcal{D}_{0}$ a topological Dirac structure. It will be shown in the next section that the singular reduced Dirac structure $\mathcal{D}_{0}$ defines a Hamiltonian formalism on the singular reduced space $M_{0}$.

In order to better comprehend the meaning of the singular reduced Dirac structure defined in Definition [18, we show that in the case of regular reduction the topological Dirac structure $\mathcal{D}_{0}$ exactly defines the regular reduced Dirac structure on $M_{0}$.

Regular reduction. Suppose that $\mu=0$ is a regular value of the momentum map and $G$ acts regularly on $M$ (that is, $M / G$ is a smooth manifold and the projection $M \rightarrow M / G$ is a surjective submersion; for example, if $G$ acts freely and properly, these conditions are satisfied). According to the results described in Section 3 the Dirac structure $\mathcal{D}$ on $M$ is reduced to a Dirac structure $\hat{\mathcal{D}}$ on the manifold $M_{0}$ in two steps: firstly, $\mathcal{D}$ is restricted to a Dirac structure $\mathcal{D}_{N}$ on $N=P^{-1}(0)$ defined by (3.4) and, secondly, $\mathcal{D}_{N}$ is projected to a Dirac structure $\hat{\mathcal{D}}$ on $M_{0}$ defined by (3.6) (with $\mathfrak{D}_{l o c}$ replaced by $\left.\left(\mathfrak{D}_{N}\right)_{l o c}\right)$. Otherwise stated, in terms of its local sections,

$$
\begin{gathered}
\hat{\mathfrak{D}}_{l o c}=\left\{(\hat{X}, \hat{\alpha}) \in \mathfrak{X}_{l o c}\left(M_{0}\right) \oplus \Omega_{l o c}^{1}\left(M_{0}\right) \mid \exists(X, \alpha) \in \mathfrak{D}_{l o c} \text { such that } X \text { is tangent to } N\right. \\
\text { and } \left.\iota_{*} X \sim_{\pi} \hat{X}, \iota^{*} \alpha=\pi^{*} \hat{\alpha}\right\},
\end{gathered}
$$

where $\iota: N \hookrightarrow M$ is the inclusion and $\iota_{*} X$ denotes the push forward of $X$ to a vector field on $N$ (that is, $\iota_{*} X$ is simply the restriction of $X$ to $N$ which is possible since $N$ is a closed submanifold of $M$ and $X$ is tangent to $N$ by hypothesis).

Consider the topological Dirac structure $\mathcal{D}_{0}$ given by Definition [18 Since $M_{0}$ is a manifold, the generalized Poisson bracket $\{\cdot, \cdot\}_{0}$ on the set of smooth functions $C^{\infty}\left(M_{0}\right)$ (see also Remark 17) defines a skew-symmetric vector bundle map $J_{0}: T^{*} M_{0} \rightarrow T M_{0}$ by $J_{0}\left(d f_{0}, d h_{0}\right)=\left\{f_{0}, h_{0}\right\}_{0}, f_{0}, h_{0} \in$ $C^{\infty}\left(M_{0}\right)$. The vector space $\hat{\Delta}$ of derivations on $C^{\infty}\left(M_{0}\right)$ defines a constant dimensional distribution of vector fields on $M_{0}$ (in other words, a vector subbundle of $T M_{0}$ ), also denoted by $\hat{\Delta}$. Then the topological Dirac structure $\mathcal{D}_{0}$ defines a Dirac structure on the manifold $M_{0}$, also denoted by $\mathcal{D}_{0}$, which in terms of its local sections is given by

$$
\left(\mathfrak{D}_{0}\right)_{l o c}=\left\{(\hat{X}, \hat{\alpha}) \in \mathfrak{X}_{l o c}\left(M_{0}\right) \oplus \Omega_{l o c}^{1}\left(M_{0}\right) \mid \hat{X}-J_{0} \hat{\alpha} \in \Gamma_{l o c}(\hat{\Delta}), \hat{\alpha} \in \Gamma_{l o c}\left(\hat{\Delta}^{\circ}\right)\right\} .
$$

Indeed, $\mathcal{D}_{0}$ is a Dirac structure on $M_{0}$ as defined in Definition 1 (notice that it is of the same form as in (2.9) ). We show that $\hat{\mathcal{D}}=\mathcal{D}_{0}$. Since both are Dirac structures and therefore their fibers are of the same dimension (i.e., $\operatorname{dim} M_{0}$ ), it is enough to show that $\hat{\mathcal{D}} \subset \mathcal{D}_{0}$. 
If $(\hat{X}, \hat{\alpha})$ is a local section of $\hat{\mathcal{D}}$, then there exists a local section $(X, \alpha)$ of $\mathcal{D}$ such that $\iota_{*} X \sim_{\pi} \hat{X}$ and $\iota^{*} \alpha=\pi^{*} \hat{\alpha}$. Since $(X, \alpha)$ is a local section of $\mathcal{D}$ one has

$$
Z:=X-J \alpha \text { is a local section of } \Delta, \alpha \text { is a local section of } \Delta^{\circ} .
$$

Consider the vector field $J \alpha \in \mathfrak{X}_{l o c}(M)$. Since $(J \alpha, \alpha) \in \mathfrak{D}_{l o c}$ it follows from (3.7) and $\mathcal{D}=\mathcal{D}^{\perp}$ that

$$
(J \alpha)\left[P_{\xi}\right](x)=\left\langle d P_{\xi}, J \alpha\right\rangle(x)=-\left\langle\alpha, \xi_{M}\right\rangle(x)=-\langle\hat{\alpha}, 0\rangle(\pi(x))=0, \forall x \in N, \forall \xi \in \mathfrak{g} .
$$

This implies that the vector field $J \alpha$ is tangent to $N$. Furthermore, by construction of the reduced generalized bracket (4.3) it follows that $\iota_{*}(J \alpha) \sim_{\pi} J_{0} \hat{\alpha}$. Since also $\iota_{*} X \sim_{\pi} \hat{X}$, equation (4.13) implies that there exists a vector field $\hat{Z} \in \mathfrak{X}_{\text {loc }}\left(M_{0}\right)$ such that $\iota_{*} Z \sim_{\pi} \hat{Z}$. It follows that $\hat{Z} \in \Gamma_{\text {loc }}(\hat{\Delta})$ by construction of $\hat{\Delta}$. This yields

$$
\hat{X}-J_{0} \hat{\alpha}=\hat{Z} \in \Gamma_{l o c}(\hat{\Delta}) .
$$

By construction, the distribution $\hat{\Delta}$ is spanned by vector fields $\hat{Z}_{1}, \ldots, \hat{Z}_{m}$ for which there exists a basis of vector fields $Z_{1}, \ldots, Z_{m} \in \Gamma_{l o c}(\Delta)$ such that $\iota_{*} Z_{j} \sim_{\pi} \hat{Z}_{j}, j=1, \ldots, m$. Since $\iota^{*} \alpha=\pi^{*} \hat{\alpha}$ and $\alpha \in \Gamma_{l o c}\left(\Delta^{\circ}\right)$, it follows immediately that

$$
\left\langle\hat{\alpha}, \hat{Z}_{j}\right\rangle \circ \pi=\left\langle\alpha, Z_{j}\right\rangle \circ \iota=0, j=1, \ldots, m,
$$

and therefore $\hat{\alpha} \in \Gamma_{l o c}\left(\hat{\Delta}^{\circ}\right)$. In conclusion, $(\hat{X}, \hat{\alpha})$ is a local section of $\mathcal{D}_{0}$. So we have shown that $\hat{\mathcal{D}} \subset \mathcal{D}_{0}$ and since both are Dirac structures on $M_{0}$ this implies that $\hat{\mathcal{D}}=\mathcal{D}_{0}$.

We conclude that in the case of regular reduction the topological Dirac structure $\mathcal{D}_{0}$ exactly defines the regular reduced Dirac structure on $M_{0}$.

\section{$5 \quad$ Singular dynamics}

In this section a Hamiltonian formalism is described corresponding to the singular reduced Dirac structure $\mathcal{D}_{0}$ of Definition [18, This formalism defines the dynamics corresponding to an implicit Hamiltonian system $\left(M_{0}, \mathcal{D}_{0}, H_{0}\right)$ on the topological space $M_{0}$. We show that if $\left(M_{0}, \mathcal{D}_{0}, H_{0}\right)$ is the reduction of the implicit Hamiltonian system $(M, \mathcal{D}, H)$ to $M_{0}$, then the $G$-projectable solutions of $(M, \mathcal{D}, H)$ project to solutions of the reduced system $\left(M_{0}, \mathcal{D}_{0}, H_{0}\right)$.

First let us define a Hamiltonian formalism on a topological space in the spirit of Sikorski differential spaces (see [26, 11]). Consider a topological space $M_{0}$ together with a subalgebra $C^{\infty}\left(M_{0}\right)$ of the continuous functions on $M_{0}$, called the set of smooth functions on $M_{0}$. A continuous curve $\gamma(t)$ on $M_{0}$ is said to be smooth (see [26]) if $f_{0} \circ \gamma$ is smooth, as a function from (a subinterval of) $\mathbb{R}$ to $\mathbb{R}$, for every $f_{0} \in C^{\infty}\left(M_{0}\right)$. Let $\hat{X}$ denote a derivation on $C^{\infty}\left(M_{0}\right)$. An integral curve of $\hat{X}$ through some point $x_{0} \in M_{0}$ is defined (see [26]) as a smooth curve $\gamma(t)$ for which, cf. (4.6),

$$
\frac{d}{d t} f_{0}(\gamma(t))=\hat{X}\left[f_{0}\right](\gamma(t)), \quad \forall t, \forall f_{0} \in C^{\infty}\left(M_{0}\right), \gamma(0)=x_{0}
$$

Let $\mathcal{D}_{0}$ be a topological Dirac structure on $M_{0}$, consisting of a generalized Poisson bracket $\{\cdot, \cdot\}_{0}$ : $C^{\infty}\left(M_{0}\right) \times C^{\infty}\left(M_{0}\right) \rightarrow C^{\infty}\left(M_{0}\right)$ (that is, the Jacobi identity does not necessarily hold) and a vector 
space $\hat{\Delta}$ of derivations on $C^{\infty}\left(M_{0}\right)$. Furthermore let $H_{0} \in C^{\infty}\left(M_{0}\right)$ be a smooth function on $M_{0}$, called the Hamiltonian function. Notice that $\left\{\cdot, H_{0}\right\}_{0}: C^{\infty}\left(M_{0}\right) \rightarrow C^{\infty}\left(M_{0}\right)$ defines a derivation on $C^{\infty}\left(M_{0}\right)$ by $\left\{\cdot, H_{0}\right\}_{0}\left[f_{0}\right]:=\left\{f_{0}, H_{0}\right\}_{0}, f_{0} \in C^{\infty}\left(M_{0}\right)$. Furthermore, if $\hat{X}$ is a derivation on $C^{\infty}\left(M_{0}\right)$ and $x \in M_{0}$, then $\hat{X}(x): C^{\infty}\left(M_{0}\right) \rightarrow \mathbb{R}$ is defined by $(\hat{X}(x))\left[f_{0}\right]:=\hat{X}\left[f_{0}\right](x), f_{0} \in C^{\infty}\left(M_{0}\right)$. The three-tuple $\left(M_{0}, \mathcal{D}_{0}, H_{0}\right)$ defines an implicit Hamiltonian system in the following way:

Definition 19. A smooth curve $\gamma(t)$ on $M_{0}$ is called an integral curve (or, solution) of $\left(M_{0}, \mathcal{D}_{0}, H_{0}\right)$ if there exists a derivation $\hat{X}$ on $C^{\infty}\left(M_{0}\right)$ such that $\gamma(t)$ is an integral curve of $\hat{X}$ and

$$
\begin{gathered}
\hat{X}(\gamma(t))-\left\{\cdot, H_{0}\right\}_{0}(\gamma(t)) \in \hat{\Delta}(\gamma(t)), \forall t, \\
\hat{Z}\left[H_{0}\right](\gamma(t))=0, \forall t, \forall \hat{Z} \in \hat{\Delta} .
\end{gathered}
$$

The implicit Hamiltonian system $\left(M_{0}, \mathcal{D}_{0}, H_{0}\right)$ is defined as the total set of integral curves $\gamma(t)$ of $\left(M_{0}, \mathcal{D}_{0}, H_{0}\right)$.

If $M_{0}$ is a smooth manifold, Definition 19 of an implicit Hamiltonian system equals Definition 6 given in Section 2 (with $\mathcal{D}_{0}$ defined by (4.12)). However, since in general $M_{0}$ is not a smooth manifold but only a topological space, the implicit Hamiltonian system $\left(M_{0}, \mathcal{D}_{0}, H_{0}\right)$ cannot be written as a set of differential and algebraic equations. As for implicit Hamiltonian systems defined on manifolds, the implicit Hamiltonian system $\left(M_{0}, \mathcal{D}_{0}, H_{0}\right)$ is energy conserving, cf. (2.13),

$$
\frac{d H_{0}}{d t}(\gamma(t))=\hat{X}\left(H_{0}\right)(\gamma(t))=\left\{H_{0}, H_{0}\right\}_{0}(\gamma(t))=0, \forall t
$$

Remark 20. Equation (5.2) implies that

$$
\frac{d}{d t} f_{0}(\gamma(t))=\left\{f_{0}, H_{0}\right\}_{0}(\gamma(t)), \forall t, \forall f_{0} \in \mathcal{A}_{\mathcal{D}_{0}},
$$

where $\mathcal{A}_{\mathcal{D}_{0}}=\left\{f_{0} \in C^{\infty}\left(M_{0}\right) \mid \hat{Z}\left[f_{0}\right]=0, \forall \hat{Z} \in \hat{\Delta}\right\}$. However, (5.5) does not imply (5.2). Even in the regular case it is not true that $\hat{Z}$ being a local section of $\hat{\Delta}$ is equivalent to $\hat{Z}\left[f_{0}\right]=0, \forall f_{0} \in \mathcal{A}_{\mathcal{D}_{0}}$. A counterexample can easily be constructed (by considering a suitable noninvolutive distribution $\hat{\Delta})$.

Remark 21. If $\hat{\Delta}=0$ (i.e., $\Delta=0$, that is, no constraints), then $\mathcal{A}_{\mathcal{D}_{0}}=C^{\infty}\left(M_{0}\right)$ and (5.2, 5.3) are equivalent to (5.5) . In this case, the Hamiltonian dynamics defined by (5.5) is exactly the singular reduced Hamiltonian dynamics as defined in [10, 9, 23, 24, 27.

Recall that implicit Hamiltonian systems defined on manifolds define a set of differential and algebraic equations and, as a consequence, the standard results on existence and uniqueness of solutions for ordinary differential equations do not apply. As explained in Section 2] in general one cannot expect neither global existence nor uniqueness of solutions of these systems. Therefore one cannot expect global existence and uniqueness of solutions of implicit Hamiltonian systems on topological spaces as defined in Definition [19. In particular, all solutions necessarily lie in the constraint space

$$
M_{0}^{c}=\left\{x \in M_{0} \mid \hat{Z}\left[H_{0}\right](x)=0, \forall \hat{Z} \in \hat{\Delta}\right\}
$$


(a topological space whose topology is induced from $\left.M_{0}\right)$. However, if $\left(M_{0}, \mathcal{D}_{0}, H_{0}\right)$ is the singular reduction of an implicit Hamiltonian system $(M, \mathcal{D}, H)$, then we shall show next that the $G$-projectable solutions of $(M, \mathcal{D}, H)$ (if they exist) project to solutions of the reduced system $\left(M_{0}, \mathcal{D}_{0}, H_{0}\right)$.

Let $x(t)$ be a solution of $(M, \mathcal{D}, H)$ with $x(0) \in N=P^{-1}(0)$. Then by (3.8), the curve $x(t)$ is contained in $N$. Now assume that $x(t)$ is a $G$-projectable solution, that is, there exists a projectable derivation (i.e., vector field) $X$ on $C^{\infty}(M)^{G}$, which projects to a well defined derivation $\hat{X}$ on $C^{\infty}\left(M_{0}\right)$, such that $x(t)$ is an integral curve of $X$ (i.e., $\left.\dot{x}(t)=X(x(t))\right)$. By (2.8) and (2.12)

$$
\begin{gathered}
X(x(t))-\{\cdot, H\}(x(t))=: Z(x(t)) \in \Delta(x(t)), \forall t, \\
Y[H](x(t))=0, \forall t, \forall Y \in \Gamma_{l o c}(\Delta) .
\end{gathered}
$$

Let $M_{0}$ be the singular reduced space and $\mathcal{D}_{0}$ be the singular reduced Dirac structure on $M_{0}$. Since $H$ is assumed to be $G$-invariant, its restriction to $N$ projects to a well defined function $H_{0} \in C^{\infty}\left(M_{0}\right)$ defined by $H_{0} \circ \pi=\left.H\right|_{N}$; for the definition of $C^{\infty}\left(M_{0}\right)$ see Definition 15] in Section 40 Define the singular reduced implicit Hamiltonian system $\left(M_{0}, \mathcal{D}_{0}, H_{0}\right)$ as in Definition [19, Project the curve $x(t)$ to $M_{0}$ to obtain the smooth curve $\gamma(t)=\pi(x(t))$ on $M_{0}$. Then $\gamma(t)$ is an integral curve of the derivation $\hat{X}$. Indeed, take an arbitrary $f_{0} \in C^{\infty}\left(M_{0}\right)$ and let $f \in C^{\infty}(M)^{G}$ be such that $f_{0} \circ \pi=\left.f\right|_{N}$. Then

$$
\frac{d}{d t} f_{0}(\gamma(t))=\frac{d}{d t} f(x(t))=X[f](x(t))=\hat{X}\left[f_{0}\right](\gamma(t)), \forall t,
$$

where we used the fact that $x(t)$ is an integral curve of $X$, cf. (4.6), and that $X$ projects to a derivation $\hat{X}$ on $C^{\infty}\left(M_{0}\right)$. Furthermore, if $Y_{1}, \ldots, Y_{m}$ is a basis of projectable local sections of $\Delta$, projecting to a basis $\hat{Y}_{1}, \ldots, \hat{Y}_{m}$ of $\hat{\Delta}$, then it follows that

$$
0=Y_{j}[H](x(t))=\hat{Y}_{j}\left[H_{0}\right](\gamma(t)), \forall t, j=1, \ldots, m,
$$

which yields equation (5.3). It remains to be proved that (5.2) is satisfied. Notice that by (4.3) the derivation $\{\cdot, H\}$ projects to a well defined derivation $\left\{\cdot, H_{0}\right\}_{0}$ on $C^{\infty}\left(M_{0}\right)$. Since also $X$ projects to a derivation $\hat{X}$ on $C^{\infty}\left(M_{0}\right)$ it follows that the derivation $Z$ projects to a well defined derivation $\hat{Z}$ on $C^{\infty}\left(M_{0}\right)$. Since $Y_{1}, \ldots, Y_{m}$ is a projectable basis of local sections of $\Delta$ it follows from (5.7) that at each point $x_{0}$ on the curve $x(t)$, one has

$$
Z\left(x_{0}\right)=c_{1} Y_{1}\left(x_{0}\right)+\cdots+c_{m} Y_{m}\left(x_{0}\right),
$$

for some constants $c_{1}, \ldots, c_{m} \in \mathbb{R}$. We claim that

$$
\hat{Z}\left(\gamma_{0}\right)=c_{1} \hat{Y}_{1}\left(\gamma_{0}\right)+\cdots+c_{m} \hat{Y}\left(\gamma_{0}\right), \gamma_{0}=\pi\left(x_{0}\right) .
$$

Indeed, take an arbitrary $f_{0} \in C^{\infty}\left(M_{0}\right)$ and let $f \in C^{\infty}(M)^{G}$ be such that $f_{0} \circ \pi=\left.f\right|_{N}$. Then

$$
\begin{aligned}
\left(\hat{Z}\left(\gamma_{0}\right)\right)\left[f_{0}\right]=\hat{Z}\left[f_{0}\right]\left(\gamma_{0}\right) & =Z[f]\left(x_{0}\right) \\
& =\left(c_{1} Y_{1}[f]+\cdots+c_{m} Y_{m}[f]\right)\left(x_{0}\right) \\
& =\left(c_{1} \hat{Y}_{1}\left[f_{0}\right]+\cdots+c_{m} \hat{Y}_{m}\left[f_{0}\right]\right)\left(\gamma_{0}\right) \\
& =\left(c_{1} \hat{Y}_{1}\left(\gamma_{0}\right)+\cdots+c_{m} \hat{Y}_{m}\left(\gamma_{0}\right)\right)\left[f_{0}\right],
\end{aligned}
$$


which yields (5.12). Since $\hat{Y}_{1}, \ldots, \hat{Y}_{m}$ forms a basis of $\hat{\Delta}$ it follows that $\hat{Z}(\gamma(t)) \in \hat{\Delta}(\gamma(t)), \forall t$. Therefore (5.2) is satisfied, which implies that $\gamma(t)$ is an integral curve of the reduced implicit Hamiltonian system $\left(M_{0}, \mathcal{D}_{0}, H_{0}\right)$. We have obtained the following

Proposition 22. Every $G$-projectable solution $x(t)$ of $(M, \mathcal{D}, H)$ with $x(0) \in P^{-1}(0)$ projects to a solution $\gamma(t)=\pi(x(t))$ of the singular reduced implicit Hamiltonian system $\left(M_{0}, \mathcal{D}_{0}, H_{0}\right)$.

Remark 23. Suppose that the implicit Hamiltonian system $(M, \mathcal{D}, H)$ is of index 1 . As remarked in Section 2, the system can be restricted to an explicit Hamiltonian system on the constraint manifold $M_{c}$ defined by a generalized Poisson bracket denoted by $\{\cdot, \cdot\}_{c}: C^{\infty}\left(M_{c}\right) \times C^{\infty}\left(M_{c}\right) \rightarrow C^{\infty}\left(M_{c}\right)$. The $G$-action leaves the manifold $M_{c}$ invariant and it follows that $G$ is a symmetry Lie group of the explicit Hamiltonian system on $M_{c}$, i.e., $L_{\xi_{M_{c}}}\{f, g\}_{c}=\left\{L_{\xi_{M_{c}}} f, g\right\}_{c}+\left\{f, L_{\xi_{M_{c}}} g\right\}_{c}, \forall f, g \in C^{\infty}\left(M_{c}\right)$ and $L_{\xi_{M_{c}}} H_{c}=0$ (where $H_{c}=\left.H\right|_{M_{c}}$ ), for all $\xi \in \mathfrak{g}$. The corresponding equivariant momentum map is given by the restriction of $P$ to $M_{c}$. Furthermore, every solution of the (restricted) system is $G$-projectable [28, 5, 6]. We can use the singular reduction theory developed in 10, 9, 23, 27, or equivalently the theory developed in this paper by considering $\Delta=0$, to reduce the system to a Hamiltonian system on the singular reduced space $\left(M_{c}\right)_{0}$. The reduced generalized Poisson bracket $\left(\{\cdot, \cdot\}_{c}\right)_{0}$ is defined analogously to (4.3). The reduced dynamics is given by equation (5.5), with $\{\cdot, \cdot\}_{0}$ replaced by $\left(\{\cdot, \cdot\}_{c}\right)_{0}$ and $\mathcal{A}_{\mathcal{D}_{0}}=C^{\infty}\left(\left(M_{c}\right)_{0}\right)$. Global existence of solutions now follows from Proposition 22. Furthermore, if the $G$-action is proper then also uniqueness of solutions of the singular reduced system can be proved [9, 27.

\section{Orbit type decomposition}

Consider a symplectic manifold $(M, \omega)$ admitting a symmetry Lie group with a corresponding momentum map. Let $M_{0}$ denote the singular reduced space and $\{\cdot, \cdot\}_{0}$ the singular reduced Poisson bracket. The singular reduced Hamiltonian dynamics is defined by equation (5.5), cf. Remark 21] In [4, 9, 10, 11, 24, 27] it is shown that the space $M_{0}$ may be decomposed into a family of symplectic manifolds, called pieces. The decomposition is by orbit type and defines a stratification of the singular reduced space $M_{0}$. Furthermore, the Hamiltonian flow corresponding to (5.5) leaves the pieces invariant and restricts to a regular Hamiltonian flow on each of the pieces. In this section we show that these results can be generalized to singular reduced implicit Hamiltonian systems. We will treat only the special class of Dirac structures for which the generalized Poisson bracket is nondegenerate.

Consider an implicit Hamiltonian system $(M, \mathcal{D}, H)$ with a Dirac structure $\mathcal{D}$ as defined in (2.8), or (4.1), where the generalized Poisson bracket is assumed to be nondegenerate and defined by a nondegenerate two-form $\omega$ on $M$, i.e., the generalized Poisson structure $J: T^{*} M \rightarrow T M$ is given by $\omega^{-1}$, that is, $\{f, h\}=\omega\left(X_{f}, X_{h}\right), f, h \in C^{\infty}(M)$, where $X_{f}$ is defined by $d f=\omega\left(X_{f}, \cdot\right)$ and analogously for $X_{h}$. Notice that we do not assume that $\omega$ is a closed two-form. This means that $\{\cdot, \cdot\}$ does not necessarily satisfy the Jacobi identity.

Let $G$ be a symmetry Lie group of $(M, \mathcal{D}, H)$ as in Proposition 9. From now on we will assume that the action of $G$ is proper. Assume that there exists an $A d^{*}$-equivariant momentum map $P: M \rightarrow \mathfrak{g}^{*}$ such that

$$
\left.d P_{\xi}=\omega\left(\xi_{M}, \cdot\right) \quad \text { (equivalently } \xi_{M}=J d P_{\xi}\right) \text { and } \quad d P_{\xi} \in \Gamma\left(\Delta^{\circ}\right), \forall \xi \in \mathfrak{g} .
$$


Notice that this implies, but is not equivalent to, (3.7).

Remark 24. Symmetry groups as described above commonly occur within the class of constrained mechanical systems as described in Example [7 Consider the distribution $\Lambda=\operatorname{ker} A^{T}(q)$ on $Q$ and let $G$ be a Lie group acting properly on the configuration space $Q$ leaving $\Lambda$ invariant, that is, $L_{\xi_{Q}} \Gamma_{l o c}(\Lambda) \subset \Gamma_{l o c}(\Lambda), \forall \xi \in \mathfrak{g}$. The action of $G$ on $Q$ lifts to an action of $G$ on $M=T^{*} Q$ as follows (recall that $\omega=d p \wedge d q$ denotes the canonical symplectic form on $M$ ): define the infinitesimal generator $\xi_{M}$ to be the Hamiltonian vector field corresponding to the function $P_{\xi}(q, p)=p^{T} \xi_{Q}(q)$, i.e., $d P_{\xi}=\omega\left(\xi_{M}, \cdot\right), \forall \xi \in \mathfrak{g}$. Now recall that $\Delta$ is defined as the image of the matrix (2.19). Then by construction, $L_{\xi_{M}} \omega=0$ and $L_{\xi_{M}} \Gamma_{l o c}(\Delta) \subset \Gamma_{l o c}(\Delta), \forall \xi \in \mathfrak{g}$, and therefore $G$ defines a symmetry group of the implicit Hamiltonian system on $M$. The $A d^{*}$-equivariant momentum map is defined by $P(q, p)(\xi)=p^{T} \xi_{Q}(q), \forall \xi \in \mathfrak{g}$, and hence satisfies the first condition in (6.1). If we assume furthermore that the symmetry group is "horizontal", i.e., $\xi_{Q} \in \operatorname{ker} A^{T}(q), \forall \xi \in \mathfrak{g}$, then also the second condition in (6.1) is satisfied.

The manifold $M$ can be decomposed into submanifolds as follows [9, 24, 27]. Let $K$ be a compact subgroup of $G$ and define $M_{(K)}$ to be the set of points in $M$ whose stabilizer group $G_{x}=\{g \in G \mid$ $\phi(g, x)=x\}$ is conjugate to $K$, i.e.,

$$
M_{(K)}=\left\{x \in M \mid \exists g \in G \text { such that } g G_{x} g^{-1}=K\right\} .
$$

Notice that since the $G$-action is assumed to be proper, every stabilizer group $G_{x}, x \in M$, is a compact subgroup of $G . M_{(K)}$ is a submanifold of $M$ called the manifold of orbit type $(K)$. On the set of compact subgroups of $G$ define an equivalence relation by saying that $\tilde{K} \sim K$ if and only if $\tilde{K}$ is conjugate to $K$. The equivalence class of $K$ is denoted by $(K)$. As $(K)$ runs over the set of equivalence classes, the manifolds $M_{(K)}$ partition $M$. Since the $G$-action is proper this partition is locally finite. This is called the orbit type decomposition of $M$.

Next we show that the image of the tangent of the momentum map at the point $x \in M$ is equal to the annihilator in $\mathfrak{g}^{*}$ of the Lie algebra of the stabilizer group $G_{x}$, i.e.,

$$
\operatorname{Im} T_{x} P=\mathfrak{g}_{x}^{\circ}, \forall x \in M,
$$

see also [1, 9, 14, 18, 24]. Indeed

$$
\xi \in \mathfrak{g}_{x} \Leftrightarrow \xi_{M}(x)=0 \Leftrightarrow d P_{\xi}(x)=0 \Leftrightarrow\left(T_{x} P(v)\right) \xi=0, \forall v \in T_{x} M \Leftrightarrow \xi \in\left(\operatorname{Im} T_{x} P\right)^{\circ},
$$

where we used (6.1) and the fact that $\omega$ is nondegenerate. This yields that $\mathfrak{g}_{x}=\left(\operatorname{Im} T_{x} P\right)^{\circ}$. Taking the annihilator of both sides yields (6.3).

Equation (6.3) implies that the tangent of the restriction of the momentum map $P$ to the manifold $M_{(K)}$ has constant rank equal to the codimension of $K$ in $G$. It follows that the intersection $P^{-1}(0) \cap M_{(K)}$ is a smooth submanifold of $M$. Furthermore, the manifold $P^{-1}(0) \cap M_{(K)}$ is invariant under the action of $G$. It turns out that the quotient $\left(M_{0}\right)_{(K)}:=\left(P^{-1}(0) \cap M_{(K)}\right) / G=$ $\pi\left(P^{-1}(0) \cap M_{(K)}\right)$ is a smooth manifold [9, 24, 27]. Consequently, the singular reduced space $M_{0}$ is decomposed into a disjoint set of manifolds, called pieces,

$$
M_{0}=\coprod_{(K)}\left(M_{0}\right)_{(K)}
$$


where $(K)$ runs over the set of conjugacy classes of compact subgroups of $G$. Since the orbit type decomposition of $M$ is locally finite, the decomposition of $M_{0}$ is also locally finite.

Next let us define a generalized Poisson bracket on each of the manifolds $\left(M_{0}\right)_{(K)}$. For clarity of exposition, consider the following commuting diagram:

$M$

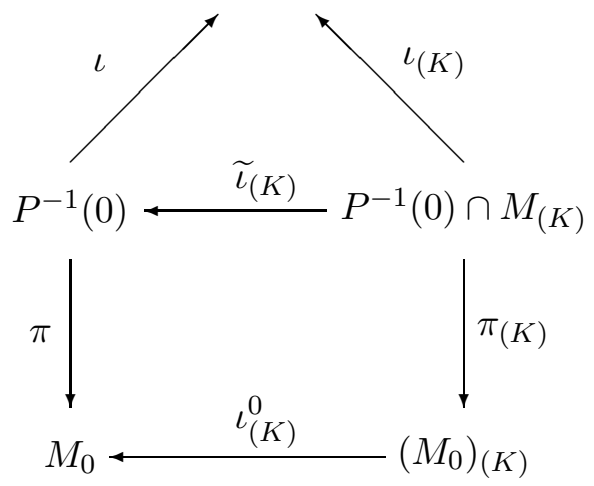

Here $\iota_{(K)}$ denotes the inclusion map and $\pi_{(K)}$ the restriction of $\pi$ to $P^{-1}(0) \cap M_{(K)}$. The inclusions $\tilde{\iota}_{(K)}$ and $\iota_{(K)}^{0}$ are defined such that the diagram commutes. Define the set of Whitney smooth functions $W^{\infty}\left(\left(M_{0}\right)_{(K)}\right)$ on $\left(M_{0}\right)_{(K)}$ as follows: a continuous function $\bar{f}_{0}$ on $\left(M_{0}\right)_{(K)}$ is said to be a Whitney smooth function if there exists a smooth $G$-invariant function $f \in C^{\infty}(M)^{G}$ such that $\bar{f}_{0} \circ \pi_{(K)}=\left.f\right|_{P^{-1}(0) \cap M_{(K)}}$. In fact, $W^{\infty}\left(\left(M_{0}\right)_{(K)}\right)$ is equal to the set of functions obtained by the restriction of the functions in $C^{\infty}\left(M_{0}\right)$ to $\left(M_{0}\right)_{(K)}$ (this is why it is called the set of Whitney smooth functions). Indeed, the $G$-invariant function $f$ descends to a smooth function $f_{0}$ on $M_{0}$, whose restriction to $\left(M_{0}\right)_{(K)}$ is precisely $\bar{f}_{0}$. This can be seen as follows:

$$
f_{0} \circ \iota_{(K)}^{0} \circ \pi_{(K)}=f_{0} \circ \pi \circ \tilde{\iota}_{(K)}=f \circ \iota \circ \tilde{\iota}_{(K)}=f \circ \iota_{(K)}=\bar{f}_{0} \circ \pi_{(K)},
$$

and since $\pi_{(K)}$ is surjective the result follows. Analogously to (4.3) define a generalized Poisson bracket on $W^{\infty}\left(\left(M_{0}\right)_{(K)}\right)$ by

$$
\left\{\bar{f}_{0}, \bar{h}_{0}\right\}_{(K)} \circ \pi_{(K)}=\left.\{f, h\}\right|_{P^{-1}(0) \cap M_{(K)}} .
$$

We need to show that the bracket is well defined and does not depend on the choice of $G$-invariant extensions $f$ and $h$. If we can prove that for every $f \in C^{\infty}(M)^{G}$ the flow of the Hamiltonian vector field $X_{f}=\{\cdot, f\}$ preserves the submanifold $P^{-1}(0) \cap M_{(K)}$, then $\mathcal{I}=\left\{f \in C^{\infty}(M)^{G} \mid\right.$ $\left.\left.f\right|_{P^{-1}(0) \cap M_{(K)}}=0\right\}$ is a Poisson ideal of $C^{\infty}(M)^{G}$ and it follows that the bracket is well defined, see e.g. 9], Appendix B, (5.2) \& (5.3). Now consider $f \in C^{\infty}(M)^{G}$, that is, $\phi_{g}^{*} f=f$, for all $g \in G$, where $\phi_{g}: M \rightarrow M$ denotes the diffeomorphism given by the action of the element $g \in G$ on $M$. Thus, since the action is symplectic, it follows that $\phi_{g}^{*} X_{f}=X_{\phi_{g}^{*} f}=X_{f}$ for all $g \in G$, which implies that the flow $\psi_{t}^{f}$ of $X_{f}$ commutes with $\phi_{g}$ for every $g \in G$. We need to show that $\psi_{t}^{f}$ preserves $M_{(K)}$. Since $M_{(K)}=G \cdot M_{K}$, where $M_{K}=\left\{x \in M \mid G_{x}=K\right\}$ (which is a submanifold of $M$ ) and the flow of $X_{f}$ and the $G$-action commute, it is enough to show that $\psi_{t}^{f}$ preserves $M_{K}$. Now, for every $g \in K$ and $x \in M_{K}$

$$
\phi_{g}\left(\psi_{t}^{f}(x)\right)=\psi_{t}^{f}\left(\phi_{g}(x)\right)=\psi_{t}^{f}(x),
$$

since $\phi_{g}(x)=x$. Therefore, $K \subset G_{\psi_{t}^{f}(x)}$ (the stabilizer group of $\psi_{t}^{f}(x)$ ). Suppose $g \in G_{\psi_{t}^{f}(x)}$, i.e., $\phi_{g}\left(\psi_{t}^{f}(x)\right)=\psi_{t}^{f}(x)$. Since the flow and the $G$-action commute, this implies that $\psi_{t}^{f}\left(\phi_{g}(x)\right)=\psi_{t}^{f}(x)$. 
Applying $\psi_{-t}^{f}$ to this relation yields $\phi_{g}(x)=x$ so $g \in K$, i.e., $G_{\psi_{t}^{f}(x)} \subset K$. It follows that $G_{\psi_{t}^{f}(x)}=K$ and therefore $\psi_{t}^{f}(x) \in M_{K}$. So we have shown that for any $G$-invariant function $f$ the flow $\psi_{t}^{f}$ preserves the submanifold $P^{-1}(0) \cap M_{(K)}$. This implies that the bracket (6.7) is well defined.

Remark 25. The set of Whitney smooth functions $W^{\infty}\left(\left(M_{0}\right)_{(K)}\right)$ is dense in the set $C^{\infty}\left(\left(M_{0}\right)_{(K)}\right)$ of smooth functions as defined by the differential structure on $\left(M_{0}\right)_{(K)}$. Indeed, the pullback to $P^{-1}(0) \cap M_{(K)}$ of a smooth function $\bar{f}_{0} \in C^{\infty}\left(\left(M_{0}\right)_{(K)}\right)$ compactly supported on $\left(M_{0}\right)_{(K)}$ can be extended to a smooth $G$-invariant function $f$ on $M$. Thus the bracket in (6.7) is a well defined generalized Poisson bracket $\{\cdot, \cdot\}_{(K)}$ on $C^{\infty}\left(\left(M_{0}\right)_{(K)}\right)$. The associated generalized Poisson structure $J_{(K)}$ is the inverse of the nondegenerate two-form $\omega_{(K)}$ defined by the condition that the pullback of $\omega_{(K)}$ to $P^{-1}(0) \cap M_{(K)}$ equals the restriction of $\omega$ to $P^{-1}(0) \cap M_{(K)}$, see also [9, 24, 27].

Having defined a generalized Poisson bracket on $\left(M_{0}\right)_{(K)}$ we now show that the distribution $\Delta$ projects to a distribution $(\hat{\Delta})_{(K)}$ on the manifold $\left(M_{0}\right)_{(K)}$. Recall from Section 4 that there exists a basis $X_{1}, \ldots, X_{m}$ of local sections of $\Delta$ such that $\left[\xi_{M}, X_{j}\right] \in \mathfrak{V}_{l o c}, \forall \xi \in \mathfrak{g}, j=1, \ldots, m$. By Proposition 9 it follows that

$$
\left[\xi_{M}, X_{j}\right] \in \Gamma_{l o c}(\Delta \cap V), \quad \forall \xi \in \mathfrak{g}, j=1, \ldots, m .
$$

If we make the assumption that $\Delta \cap V=0$, then it follows that $\left[\xi_{M}, X_{j}\right]=0, \forall \xi \in \mathfrak{g}, j=1, \ldots, m$. This means that the flow of $X_{j}$ commutes with the $G$-action and therefore preserves the submanifold $P^{-1}(0) \cap M_{(K)}$. This implies that $X_{j}$ is tangent to $P^{-1}(0) \cap M_{(K)}$ and restricts to a local vector field $\bar{X}_{j}$ on $P^{-1}(0) \cap M_{(K)}$, for all $j=1, \ldots, m$. The basis $\bar{X}_{1}, \ldots, \bar{X}_{m}$ projects to a set of independent local vector fields $\hat{X}_{1}, \ldots, \hat{X}_{m}$ on $\left(M_{0}\right)_{(K)}$ which define a distribution $(\hat{\Delta})_{(K)}$ on $\left(M_{0}\right)_{(K)}$.

Remark 26. If the system is of index 1 , then it is always true that $\Delta(x) \cap V(x)=0, \forall x \in M_{c}$ (see [5, 6]).

The generalized Poisson bracket $\{\cdot, \cdot\}_{(K)}$, with its generalized Poisson structure $J_{(K)}$, and the distribution $(\hat{\Delta})_{(K)}$ define a Dirac structure $\mathcal{D}_{(K)}$ on $\left(M_{0}\right)_{(K)}$, given in terms of its local sections by

$$
\begin{aligned}
\left(\mathfrak{D}_{(K)}\right)_{l o c}=\{ & (\hat{X}, \hat{\alpha}) \in \mathfrak{X}_{l o c}\left(\left(M_{0}\right)_{(K)}\right) \times \Omega_{l o c}^{1}\left(\left(M_{0}\right)_{(K)}\right) \mid \\
& \left.\hat{X}-J_{(K)} \hat{\alpha} \in \Gamma_{l o c}\left((\hat{\Delta})_{(K)}\right), \hat{\alpha} \in \Gamma_{l o c}\left(\left((\hat{\Delta})_{(K)}\right)^{\circ}\right)\right\} .
\end{aligned}
$$

The results in Section 4 imply that $\mathcal{D}_{(K)}$ is exactly the regular reduced Dirac structure on $\left(M_{0}\right)_{(K)}$. We summarize the previous discussion in the following statement.

Proposition 27. Consider an implicit Hamiltonian system $(M, \mathcal{D}, H)$ with a Dirac structure $\mathcal{D}$ of the type given in (2.8), defined by a nondegenerate generalized Poisson bracket $\{\cdot, \cdot\}$ and a smooth vector subbundle $\Delta$ of TM. Assume the system admits a symmetry Lie group G acting properly on $M$ and satisfying the hypotheses of Proposition [9. Suppose also that the action admits an Ad $^{*}$ equivariant momentum map $P$ satisfying [6.1). Assume furthermore that $\Delta \cap V=0$ and that the assumptions in Propositions 10 and 13 are satisfied. ${ }^{8}$ Then the singular reduced space $M_{0}$ is

\footnotetext{
${ }^{8}$ Notice that $\Delta$ and $V$ being constant dimensional distributions and $\Delta \cap V=0$ imply that $\Delta+V$ is a constant dimensional distribution, i.e., a smooth vector subbundle of $T M$.
} 
decomposed into a disjoint set of manifolds $\left(M_{0}\right)_{(K)}$, cf. (6.5), called pieces. The system $(M, \mathcal{D}, H)$ reduces, by means of regular reduction, to an implicit Hamiltonian system $\left(\left(M_{0}\right)_{(K)}, \mathcal{D}_{(K)}, H_{(K)}\right)$ on each of the pieces. Here $\mathcal{D}_{(K)}$ is the Dirac structure defined in (6.10) and $H_{(K)} \in C^{\infty}\left(\left(M_{0}\right)_{(K)}\right)$ is defined by $H_{(K)} \circ \pi_{(K)}=\left.H\right|_{P^{-1}(0) \cap M_{(K)}}$.

Remark 28. The only reason why we assumed that the Dirac structure $\mathcal{D}$ is defined by a nondegenerate generalized Poisson bracket and the momentum map $P$ satisfies (6.1), is to show the equality (6.3). This equality implies that the subsets $P^{-1}(0) \cap M_{(K)}$ are smooth manifolds and consequently the pieces $\left(M_{0}\right)_{(K)}$ are smooth manifolds as well. The construction of the reduced Dirac structure $\mathcal{D}_{(K)}$ however is completely general. This means that the result of Proposition 27 is valid for general Dirac structures and $A d^{*}$-equivariant momentum maps of the form (3.7), as long as it is certain that the pieces $\left(M_{0}\right)_{(K)}$ are smooth manifolds. The same will hold for the results in the remaining part of this section. ${ }^{9}$

Next we show that the regular reduced implicit Hamiltonian systems $\left(\left(M_{0}\right)_{(K)}, \mathcal{D}_{(K)}, H_{(K)}\right)$ are precisely the restriction of the singular reduced implicit Hamiltonian system $\left(M_{0}, \mathcal{D}_{0}, H_{0}\right)$ to the pieces $\left(M_{0}\right)_{(K)}$. Indeed, first we show that the inclusion $\iota_{(K)}^{0}:\left(M_{0}\right)_{(K)} \rightarrow M_{0}$ is a Poisson map. Consider $f_{0}, h_{0} \in C^{\infty}\left(M_{0}\right)$ and their restrictions $\bar{f}_{0}, \bar{h}_{0} \in W^{\infty}\left(\left(M_{0}\right)_{(K)}\right)$ and let $f, h \in C^{\infty}(M)^{G}$ be such that $f_{0} \circ \pi=\left.f\right|_{P^{-1}(0)}$, and analogously for $h$. The definition of the brackets and the commutativity of the diagram implies that (notice that by (6.6) $\bar{f}_{0} \circ \pi_{(K)}=\left.f\right|_{P^{-1}(0) \cap M_{(K)}}$ and analogously for $\bar{h}_{0}$ )

$$
\left\{\bar{f}_{0}, \bar{h}_{0}\right\}_{(K)} \circ \pi_{(K)}=\{f, h\} \circ \iota_{(K)}=\{f, h\} \circ \iota \circ \tilde{\iota}_{(K)}=\left\{f_{0}, h_{0}\right\}_{0} \circ \pi \circ \tilde{\iota}_{(K)}=\left\{f_{0}, h_{0}\right\}_{0} \circ \iota_{(K)}^{0} \circ \pi_{(K)} .
$$

Since $\pi_{(K)}$ is surjective it follows that

$$
\left\{\bar{f}_{0}, \bar{h}_{0}\right\}_{(K)}=\left.\left\{f_{0}, h_{0}\right\}_{0}\right|_{\left(M_{0}\right)_{(K)}} .
$$

By construction, it is immediately clear that $(\hat{\Delta})_{(K)}$ is the restriction of $\hat{\Delta}$ to $W^{\infty}\left(\left(M_{0}\right)_{(K)}\right)$, defining by denseness a distribution on $C^{\infty}\left(\left(M_{0}\right)_{(K)}\right)$. We conclude that the regular reduced Dirac structure $\mathcal{D}_{(K)}$ is precisely the restriction of the singular reduced Dirac structure $\mathcal{D}_{0}$ to the piece $\left(M_{0}\right)_{(K)}$. Next, consider a solution $\gamma(t)$ of $\left(M_{0}, \mathcal{D}_{0}, H_{0}\right)$, as defined in Definition 19. Since $\hat{X}_{1}, \ldots, \hat{X}_{m}$ is a basis of local sections of $\hat{\Delta}$ there exist local functions $c_{0}^{1}, \ldots, c_{0}^{m} \in C^{\infty}\left(M_{0}\right)$ such that

$$
\hat{X}(\gamma(t))-\left\{\cdot, H_{0}\right\}_{0}(\gamma(t))=c_{0}^{1}(\gamma(t)) \hat{X}_{1}(\gamma(t))+\ldots+c_{0}^{m}(\gamma(t)) \hat{X}_{m}(\gamma(t)) \in \hat{\Delta}(\gamma(t)), \forall t .
$$

Consider the derivation

$$
\hat{Y}=\left\{\cdot, H_{0}\right\}_{0}+c_{0}^{1} \hat{X}_{1}+\ldots+c_{0}^{m} \hat{X}_{m}
$$

on $C^{\infty}\left(M_{0}\right)$. This is the projection of a local vector field $Y=\{\cdot, H\}+c^{1} X_{1}+\ldots+c^{m} X_{m}$ on $M$, where $c^{j} \in C^{\infty}(M)^{G}$ are such that $c_{0}^{j} \circ \pi=\left.c^{j}\right|_{P^{-1}(0)}$ and $\left\{X_{1}, \ldots, X_{m}\right\}$ denotes the projectable local basis of $\Delta$. Since the flow of this vector field commutes with the $G$-action, it preserves the submanifold $P^{-1}(0) \cap M_{(K)}$ (following the argument above Remark 25). It follows that the flow corresponding to the integral curve $\gamma(t)$ preserves the pieces $\left(M_{0}\right)_{(K)}$ and therefore $\gamma(t)$ restricts to

\footnotetext{
${ }^{9}$ One could even think of further generalizing the results by allowing the pieces to be topological spaces instead of manifolds.
} 
a smooth curve $\bar{\gamma}(t)$ on $\left(M_{0}\right)_{(K)}$. The vector field $Y$ restricts to a vector field $\hat{Y}^{\prime}$ on $\left(M_{0}\right)_{(K)}$. By construction it follows that

$$
\hat{Y}^{\prime}=\left\{\cdot, H_{(K)}\right\}_{(K)}+\bar{c}_{0}^{1} \hat{X}_{1}^{\prime}+\ldots+\bar{c}_{0}^{m} \hat{X}_{m}^{\prime}
$$

where $\bar{c}_{0}^{j}=\left.c_{0}^{j}\right|_{\left(M_{0}\right)_{(K)}}$ and $\hat{X}_{j}^{\prime}$ is the restriction of $\hat{X}_{j}$ to $\left(M_{0}\right)_{(K)}, j=1, \ldots, m$. The curve $\bar{\gamma}(t)$ is an integral curve of $\hat{Y}^{\prime}$. Indeed,

$$
\frac{d}{d t} \bar{f}_{0}(\bar{\gamma}(t))=\frac{d}{d t} f_{0}(\gamma(t))=\hat{X}\left[f_{0}\right](\gamma(t))=\hat{Y}\left[f_{0}\right](\gamma(t))=\hat{Y}^{\prime}\left[\bar{f}_{0}\right](\bar{\gamma}(t)), \forall \bar{f}_{0} \in W^{\infty}\left(\left(M_{0}\right)_{(K)}\right),
$$

and since $W^{\infty}\left(\left(M_{0}\right)_{(K)}\right)$ is dense in $C^{\infty}\left(\left(M_{0}\right)_{(K)}\right)$ the result follows. Furthermore, it is clear that

$$
\hat{Y}^{\prime}(\bar{\gamma}(t))-J_{(K)}(\bar{\gamma}(t)) d H_{(K)}(\bar{\gamma}(t))=\hat{Y}^{\prime}(\bar{\gamma}(t))-\left\{\cdot, H_{(K)}\right\}_{(K)}(\bar{\gamma}(t)) \in \Gamma_{l o c}\left((\hat{\Delta})_{(K)}\right)(\bar{\gamma}(t)), \forall t
$$

and

$$
\hat{Z}^{\prime}\left[H_{(K)}\right](\bar{\gamma}(t))=0, \forall t, \forall \hat{Z}^{\prime} \in \Gamma_{l o c}\left((\hat{\Delta})_{(K)}\right),
$$

which means that $\bar{\gamma}(t)$ is a solution of the regular reduced Hamiltonian system $\left(\left(M_{0}\right)_{(K)}, \mathcal{D}_{(K)}, H_{(K)}\right)$. In conclusion we have proved the following:

Proposition 29. Consider the conditions in Proposition 27. The regular reduced implicit Hamiltonian systems $\left(\left(M_{0}\right)_{(K)}, \mathcal{D}_{(K)}, H_{(K)}\right)$ are exactly the restrictions of the singular reduced implicit Hamiltonian system $\left(M_{0}, \mathcal{D}_{0}, H_{0}\right)$ to the pieces $\left(M_{0}\right)_{(K)}$. A solution $\gamma(t)$, with $\gamma(0) \in\left(M_{0}\right)_{(K)}$, of $\left(M_{0}, \mathcal{D}_{0}, H_{0}\right)$ preserves the piece $\left(M_{0}\right)_{(K)}$ and restricts to a solution $\bar{\gamma}(t)$ of $\left(\left(M_{0}\right)_{(K)}, \mathcal{D}_{(K)}, H_{(K)}\right)$.

Finally, remark that since the pieces $\left(M_{0}\right)_{(K)}$ are smooth manifolds, each implicit Hamiltonian system $\left(\left(M_{0}\right)_{(K)}, \mathcal{D}_{(K)}, H_{(K)}\right)$ can be written as a set of differential and algebraic equations (DAE). The singular reduced implicit Hamiltonian system $\left(M_{0}, \mathcal{D}_{0}, H_{0}\right)$ can thus be written as a collection of DAEs, one (set of differential and algebraic equations) on each piece.

\section{Conclusions}

In this paper we studied the singular reduction of implicit Hamiltonian systems admitting a symmetry Lie group with a corresponding equivariant momentum map. The results extend the singular reduction theory known for explicit symplectic or Poisson Hamiltonian systems [3, 4, 9, 10, 11, 23, 24, 27. The main result is a purely topological description of the reduced implicit Hamiltonian system using the definition of a topological Dirac structure. In particular, the reduced space is not assumed to be a smooth manifold. The dynamics corresponding to this system are defined and it is shown that the projectable solutions of the unreduced system project to solutions of the reduced system. If the symmetry Lie group acts freely and properly and the value of the momentum map is regular, then the singular reduced implicit Hamiltonian system equals the regular reduced implicit Hamiltonian system as described in [5, 6]. Finally, under certain conditions, the singular reduced space can be decomposed into a set of smooth manifolds called pieces. It is shown that the singular reduced implicit Hamiltonian system restricts to regular reduced implicit Hamiltonian systems on all the pieces. 
Acknowledgments. This research was performed during the time when the first author held a postdoctoral position at EPFL, Switzerland. G.B. gratefully acknowledges the hospitality and the financial support of this institution. Furthermore, G.B. also acknowledges the financial support received from the European sponsored project GeoPlex (IST-2001-34166, www.geoplex.cc). T.S.R. was partially supported by the European Commission and the Swiss Federal Government through funding for the Research Training Network Mechanics and Symmetry in Europe (MASIE) as well as the Swiss National Science Foundation.

\section{References}

[1] R. Abraham and J.E. Marsden. Foundations of Mechanics. Benjamin / Cummings Publishing Company, second edition, 1978.

[2] R. Abraham, J.E. Marsden, and T.S. Ratiu. Manifolds, Tensor Analysis, and Applications. Springer-Verlag, second edition, 1988.

[3] J. Arms, R. Cushman, and M. Gotay. A universal reduction procedure for Hamiltonian group actions. In T. Ratiu, editor, The Geometry of Hamiltonian Systems, pages 33-51. Springer Verlag, 1991.

[4] L. Bates and E. Lerman. Proper group actions and symplectic stratified spaces. Pacific Journal of Mathematics, 181(2):201-229, 1997.

[5] G. Blankenstein. Implicit Hamiltonian Systems: Symmetry and Interconnection. Ph.D. Thesis, University of Twente, The Netherlands, November 2000.

[6] G. Blankenstein and A.J. van der Schaft. Symmetry and reduction in implicit generalized Hamiltonian systems. Rep. Math. Phys., 47(1):57-100, 2001.

[7] A.M. Bloch and P.E. Crouch. Representations of Dirac structures on vector spaces and nonlinear L-C circuits. In H. Hermes, G. Ferraya, R. Gardner, and H. Sussmann, editors, Proc. Symp. Pure Math., Differential Geometry and Control Theory, volume 64, pages 103-117, 1999.

[8] T. Courant. Dirac manifolds. Trans. American Math. Soc., 319:631-661, 1990.

[9] R.H. Cushman and L.M. Bates. Global Aspects of Classical Integrable Systems. Birkhäuser Verlag, 1997.

[10] R. Cushman and R. Sjamaar. On singular reduction of Hamiltonian spaces. In P. Donato et al., editors, Symplectic Geometry and Mathematical Physics, pages 114-128. Birkhäuser, Boston, 1991.

[11] R. Cushman and J. Śniatycki. Differential structure of orbit spaces. Canad. J. Math., 53:715$755,2001$.

[12] M. Dalsmo and A.J. van der Schaft. On representations and integrability of mathematical structures in energy-conserving physical systems. SIAM J. Opt. Cont., 37(1):54-91, 1999. 
[13] I. Dorfman. Dirac Structures and Integrability of Nonlinear Evolution Equations. Chichester: John Wiley, 1993.

[14] P. Libermann and C.-M. Marle. Symplectic Geometry and Analytical Mechanics. Reidel, Dordrecht, 1987.

[15] Z.-J. Liu, A. Weinstein and P. Xu. Dirac structures and Poisson homogeneous spaces. Commun. Math. Phys., 192:121-144, 1998.

[16] J.E. Marsden. Lectures on Mechanics. Cambridge University Press, 1992.

[17] J.E. Marsden and T.S. Ratiu. Reduction of Poisson manifolds. Lett. in Math. Phys., 11:161$169,1986$.

[18] J.E. Marsden and T.S. Ratiu. Introduction to Mechanics and Symmetry. Springer-Verlag, second edition, 1999.

[19] J.E. Marsden and A. Weinstein. Reduction of symplectic manifolds with symmetry. Rep. Math. Phys., 5:121-130, 1974.

[20] B.M. Maschke and A.J. van der Schaft. Interconnected mechanical systems, part II: the dynamics of spatial mechanical networks. In A. Astolfi, D.J.N. Limebeer, C. Melchiorri, A. Tornambè, and R.B. Vinter, editors, Modelling and Control of Mechanical Systems, pages 17-30. Imperial College Press, 1997.

[21] H. Nijmeijer and A.J. van der Schaft. Nonlinear Dynamical Control Systems. Springer-Verlag, 1990.

[22] P.J. Olver. Applications of Lie Groups to Differential Equations. Springer-Verlag, second edition, 1993.

[23] J.-P. Ortega and T.S. Ratiu. Singular reduction of Poisson manifolds. Letters in Mathematical Physics, 46:359-372, 1998.

[24] J.-P. Ortega and T.S. Ratiu. Momentum Maps and Hamiltonian Reduction. Progress in Mathematics, Birkhäuser, Boston 2003, to appear.

[25] G.W. Schwarz. Smooth functions invariant under the action of a compact Lie group. Topology, 14:63-68. 1974.

[26] R. Sikorski. Abstract covariant derivative. Colloquium Math., 18:251-272, 1967.

[27] R. Sjamaar and E. Lerman. Stratified symplectic spaces and reduction. Annals of Mathematics, 134:375-422, 1991.

[28] A.J. van der Schaft. Implicit Hamiltonian systems with symmetry. Rep. Math. Phys., 41:203$221,1998$.

[29] A.J. van der Schaft. $L_{2}$-Gain and Passivity Techniques in Nonlinear Control. Springer-Verlag, second revised and enlarged edition, 2000. 
[30] A.J. van der Schaft and B.M. Maschke. On the Hamiltonian formulation of nonholonomic mechanical systems. Rep. Math. Phys., 34(2):225-232, 1994.

[31] A.J. van der Schaft and B.M Maschke. The Hamiltonian formulation of energy conserving physical systems with external ports. Archiv für Elektronik und Übertragungstechnik, 49:362371, 1995.

[32] A.J. van der Schaft and B.M. Maschke. Mathematical modeling of constrained Hamiltonian systems. In Preprints of the 3rd Nonlinear Control Systems Design Symposium, NOLCOS, Tahoe City, CA, 1995.

[33] A.J. van der Schaft and B.M Maschke. Interconnected mechanical systems, part I: geometry of interconnection and implicit Hamiltonian systems. In A. Astolfi, D.J.N Limebeer, C. Melchiorri, A. Tornambè, and R.B. Vinter, editors, Modelling and Control of Mechanical Systems, pages 1-15. Imperial College Press, 1997. 